\title{
Injectable non-immunogenic PEG-like conjugate that forms a subcutaneous depot and enables sustained delivery of a peptide drug
}

Imran Ozer

Duke University

Anna Slezak

Duke University

Jeffrey Everitt

Duke University https://orcid.org/0000-0003-0273-6284

Xinghai Li

Duke University

Nikita Zakharov

Duke University

Joel Collier

Duke University https://orcid.org/0000-0003-3536-4827

Jonathan Campbell

https://orcid.org/0000-0003-4358-6331

David D'Alessio

Duke Molecular Physiology Institute https://orcid.org/0000-0003-4155-4870

Ashutosh Chilkoti ( $\square$ chilkoti@duke.edu )

Duke University https://orcid.org/0000-0003-1301-1836

Article

Keywords: polyethylene glycol (PEG), biologics, drug delivery

Posted Date: July 28th, 2021

DOl: https://doi.org/10.21203/rs.3.rs-737658/v1

License: (c) (i) This work is licensed under a Creative Commons Attribution 4.0 International License.

Read Full License 

Imran Ozer ${ }^{1}$, Anna Slezak ${ }^{2+}$, Jeffrey I. Everitt ${ }^{3}$, Xinghai Li ${ }^{1}$, Nikita Zakharov ${ }^{1}$, Joel H. Collier ${ }^{1}$, Jonathan E. Campbell ${ }^{4,5,6}$, David A. D'Alessio ${ }^{4,5}$, and Ashutosh Chilkoti ${ }^{{ }^{*}}$

${ }^{1}$ Department of Biomedical Engineering, Duke University, Durham, North Carolina 27708, USA.

${ }^{2}$ Department of Chemistry, Duke University, Durham, North Carolina 27708, USA.

\section{Abstract}

14 Many biologics have a short plasma half-life, and their conjugation to polyethylene glycol (PEG) is commonly used 15 to solve this problem. Unfortunately, PEG is immunogenic and forms vacuoles, and improvement in PEGylated drugs' half-life is at an asymptote. Here, we developed a PEG-like, non-immunogenic, and injectable conjugate

17 technology for sustained delivery of biologics. An optimal poly[oligo(ethylene glycol) methyl ether methacrylate]

18 (POEGMA) depot of exendin, a peptide drug used in the clinic in treating type 2 diabetes, outperformed PEG, nondepot-forming POEGMA, and a clinical sustained-release exendin-4 formulation in efficacy and pharmacokinetics. Notably, POEGMA was non-immunoreactive, while PEG induced a persistent anti-PEG immune response, leading

21 to its subsequent doses' early clearance and loss of efficacy. POEGMA did not induce vacuolization. Solving these 
22 problems of PEG and improving upon its half-life benefits by creating injectable POEGMA conjugates that form a

23 drug depot under the skin and provide sustained efficacy breathe new life into an established and valuable drug

24 delivery technology that is facing an impasse.

25 
Introduction

Biologics are potent, highly specific, and well-tolerated and have become an important class of drugs. ${ }^{1}$ Despite their promise, most of them have a short half-life due to rapid renal elimination and in vivo degradation. ${ }^{2}$

29 Their short in vivo half-life hence necessitates frequent injections, resulting in a peak-and-valley profile in drug concentration that is pharmacologically suboptimal. It also incurs a high treatment cost and suboptimal patient

31 compliance because of the need for frequent drug administration and the side effects associate with a peak-and-

32 valley drug concentration profile. ${ }^{3,4}$ One of the most common approaches to overcome these challenges is the covalent attachment of biologics to polyethylene glycol (PEG), a technology that is colloquially termed

34 PEGylation. ${ }^{5}$ PEGylated drug conjugates have a much longer plasma half-life than the native drug due to their larger size leading to slower renal clearance, reduced opsonization that reduces clearance by the reticuloendothelial system (RES), and improved solubility. ${ }^{6}$ Unfortunately, PEGylation has several significant

37 limitations, some of which have come to the forefront recently. First, PEG was initially believed to be nonimmunogenic. However, it is now well accepted that PEGylated therapeutics induce PEG antibodies upon treatment. ${ }^{7,8}$ Pre-existing PEG antibodies have also been reported in up to $70 \%$ of the population who are naïve to PEGylated therapeutics, possibly due to chronic exposure to PEG in consumer products and because PEG is

41 used as an excipient in many drug formulations. ${ }^{9-11}$ Both induced and pre-existing PEG antibodies have caused a 42 severe allergic reaction and accelerated clearance in some patients, reducing the drugs' clinical efficacy. ${ }^{7,8,12,13}$

43 These issues have collectively led to the early termination of several clinical trials of PEGylated drug candidates 44 and the withdrawal of several PEGylated therapeutics from the market. ${ }^{12,14,15}$ Second, attempts to improve the 45 pharmacokinetics (PK) of PEG have focused on synthesizing branched and star-shaped PEGs. However, these 46 architectures have a modest effect on $\mathrm{PK}^{16}$ and have antigenic and immunogenic profiles similar to linear PEG. ${ }^{12}$ 47 Third, PEG forms vacuoles in major organs due to its non-biodegradable structure and clearance by the RES. ${ }^{17}$ 48 Clearly, optimization of PEG is now at an asymptote, and new architectures that radically depart from linear PEG 49 are needed to address these limitations. 
Motivated by these needs, we have developed a "next-gen" PEG-like polymer, poly[oligo(ethylene glycol) methyl ether methacrylate] (POEGMA). POEGMA is an amphiphilic, hyperbranched polymer that breaks up the long ethylene glycol sequences in PEG and presents them as much shorter oligomeric ethylene glycol (OEG) sidechains along a hydrophobic backbone. It has been previously shown that POEGMA with three EG unit long side chains improves the PK of exendin-4, a peptide drug used in the clinic to treat type 2 diabetes (T2D) and

55 subsequently referred to as exendin. ${ }^{18}$ The POEGMA conjugate of exendin (Ex-POEGMA) also showed no in vitro binding to human-derived PEG antibodies, presumably because the short OEG side-chains in POEGMA lack the anti-PEG epitope. ${ }^{18,19}$ Although these results were encouraging, the soluble Ex-POEGMA conjugate reported previously only showed a modest improvement in the PK of the drug. This study also did not compare the PK and efficacy of POEGMA conjugates with PEG conjugates, nor did it report the intrinsic immunogenicity of POEGMA that could occur upon repeated administration of POEGMA-drug conjugates, which are critical for its clinical 61 translation.

This paper has two goals. The first goal was to devise a strategy to further enhance the half-life of exendin

63 beyond the four days of therapeutic action of a soluble POEGMA conjugate in mice ${ }^{18}$ and do so without 64 compromising its lack of reactivity to pre-existing PEG antibodies. Improving the PK of POEGMA beyond the 65 duration exhibited by soluble POEGMA conjugates is important because recent long-acting delivery technologies such as Fc fusions have been shown to work for 4-6 days in mice ${ }^{20,21}$ and 7 days in humans ${ }^{21}$ so that a next67 generation PEGylation technology must match - at a minimum - the performance of Fc conjugates. To address 68 this goal, we report on the design and optimization of a gel-like injectable depot of an Ex-POEGMA conjugate that 69 achieves sustained release from the depot into the bloodstream while retaining its lack of reactivity towards PEG antibodies. We identify an optimal Ex-POEGMA depot that maximizes blood glucose control in hyperglycemic mice

71 and investigate its PK and efficacy benefits. The second goal was to investigate the intrinsic immunogenicity and 72 toxicity of a POEGMA-drug conjugate and compare it to PEG. We report the remarkable result that Ex-POEGMA 73 conjugates have very little if any humoral immunogenicity in mice, as seen by the lack of an immunoglobulin (Ig) 
$\mathrm{M}$ and IgG response to repeated doses of Ex-POEGMA. Furthermore, this lack of immunogenicity is highly robust, as confirmed by the lack of IgG and IgM response against POEGMA observed with a POEGMA conjugate of the highly immunogenic ovalbumin (OVA) protein, even with co-administered with an adjuvant. Finally, the optimized POEGMA conjugate did not cause the cellular vacuolization reported with conventional PEG conjugates.

\section{Results}

Injectable and depot forming POEGMA library. POEGMA exhibits lower critical solution temperature (LCST) phase

80 behavior, allowing it to transition between an aqueous soluble and insoluble coacervate phase in a temperature-

81 and concentration-dependent manner. ${ }^{22-24}$ We exploited this feature to design a drug depot under the skin and

82 achieve sustained drug release from the POEGMA depot into circulation. To do so, we first identified POEGMAs

83 that would be suitable as an injectable depot by creating a set of POEGMAs with azide-end groups (Supplementary

84 Fig. 1-2) that phase transition between room temperature $\left(25^{\circ} \mathrm{C}\right)$ and subcutaneous (s.c.) temperature of mice $\left(34^{\circ} \mathrm{C}\right)^{25}$ using activator-regenerated by electron transfer atom transfer radical polymerization (ARGETATRP). ${ }^{26}$ We restricted the EG side-chain length to $\leq 3$ (Supplementary Fig. 3-6) because previous studies showed that POEGMA conjugates with a side-chain length of $>3$ had a low, though measurable level of binding with PEG antibodies. ${ }^{18,19}$ We also fortuitously found that the phase transition temperature $\left(T_{t}\right)$ of POEGMA was sensitive to the OEG side chain length, with shorter side-chains having a lower $T_{t}$ due to the decreased hydrogen bonding. The

$90 T_{t}$ of a homopolymer POEGMA with 3EG-long side chains (EG3) was too high to form an s.c. depot, while the $T_{t}$ of

91 an EG2 homopolymer was too low, preventing it from being injected in a soluble form at room temperature.

These observations led us to hypothesize that a random copolymer of POEGMA with EG3 and EG2 units should have a $T_{t}$ that is between room temperature and body temperature across a range of concentrations

94 relevant for drug delivery, which would be ideal for the design of a conjugate that is injectable, but phase 95 transitions into an insoluble coacervate upon s.c. injection, thereby forming a drug depot. To identify a copolymer 96 with an optimal $T_{t}$ for an injectable s.c. depot, we synthesized a set of copolymers using EG2 and EG3 monomers 97 at various ratios in which the molar percentage of EG2 ranged from 58-100\%, as determined by Nuclear Magnetic 
Resonance (NMR) spectroscopy (Supplementary Fig. 7-8). ${ }^{27}$ We also synthesized an EG3 POEGMA homopolymer

99 as a non-depot forming - soluble- control. The monomer composition of POEGMA was defined as the 100 percentage of EG2 (or EG3) monomer content in the total polymer. We used the nomenclature of EGX $X_{\%}$, where $\mathrm{X}$ 101 is the length of the EG chain (2 or 3), and the subscript \% is the percentage of the monomer in the total polymer. 102 All synthesized polymers were relatively monodisperse, as measured by gel permeation chromatography-multi103 angle light scattering (GPC-MALS), with a polydispersity $(\Theta)$ of <1.2 (Fig. 1a; Supplementary Fig. 9). Their degree 104 of polymerization (DP) ranged from 100 to 400 , translating to a weight-averaged molecular weight $\left(M_{\mathrm{w}}\right)$ range of 105 20-80 kDa. Each polymer's monomer composition, $M_{\mathrm{w}}$, number-averaged molecular weight $\left(M_{\mathrm{n}}\right), \oplus, \mathrm{DP}$, 106 hydrodynamic size $\left(R_{h}\right)$, and $T_{t}$ are shown in Supplementary Fig. 7-10 and summarized in Supplementary Table 1.

All polymers showed sharp and thermally reversible phase behavior, as seen by the sharp increase in optical density as the temperature was increased and by the fact that their optical density decreased back to 109 baseline as the temperature was decreased below the $T_{t}$ (Fig. 1b). Their phase behavior also showed no thermal 110 hysteresis, as seen by the overlapping turbidity curves for the heating and cooling cycle. The EG3 ${ }_{100 \%}$ POEGMA 111 had a $\mathrm{T}_{\mathrm{t}}$ of $\sim 48 \mathrm{o} \mathrm{C}$ at $25 \mu \mathrm{M}$, consistent with a previous report, ${ }^{28}$ confirming that it cannot form an s.c. depot. The $112 T_{t}$ of the POEGMA copolymers was a function of the EG2 content, as the $T_{t}$ decreased with the increasing molar 113 ratio of the more hydrophobic EG2 monomer. All copolymers phase transitioned between 25 and 34 으, suggesting 114 that all copolymers were potential depot-forming constructs. The $T_{t}$ of POEGMA also showed an inverse 115 concentration dependence. The $T_{t}$ increased with POEGMA dilution (Fig. 1c), suggesting that sustained release of 116 a POEGMA conjugate from the s.c. depot should be possible in vivo, in response to continuous dilution at the 117 periphery of the depot. The $T_{t}$ of POEGMA was also a function of its $M_{w}$, as the $T_{t}$ decreased with increasing DP118 and $M_{\mathrm{w}}$ (Fig. 1d). These data clearly showed that the key molecular variables that control the phase behavior of 119 POEGMA copolymers - namely its $\mathrm{T}_{\mathrm{t}}$ - are the composition - the ratio of EG2 to EG3 units - and $M_{\mathrm{w}}$. 
a

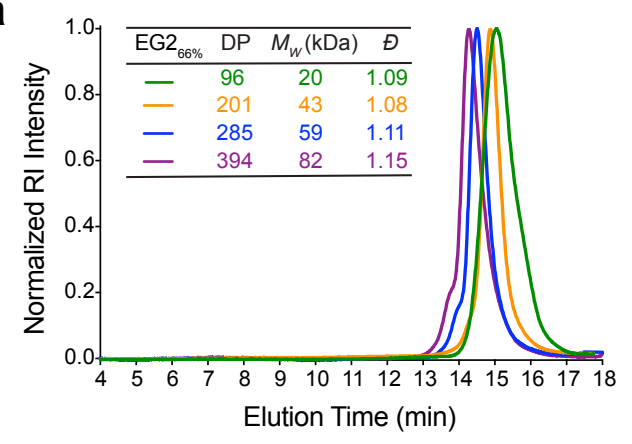

C

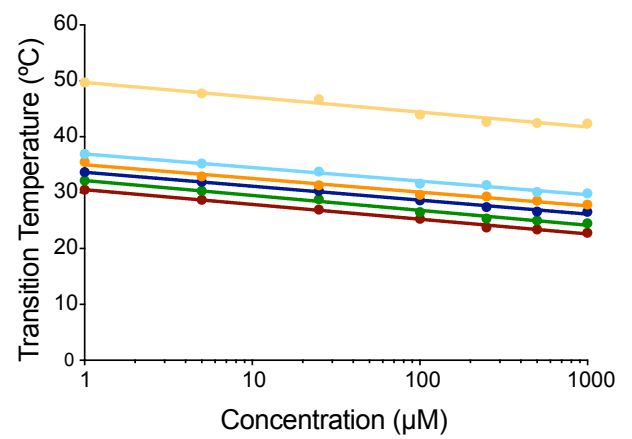

b

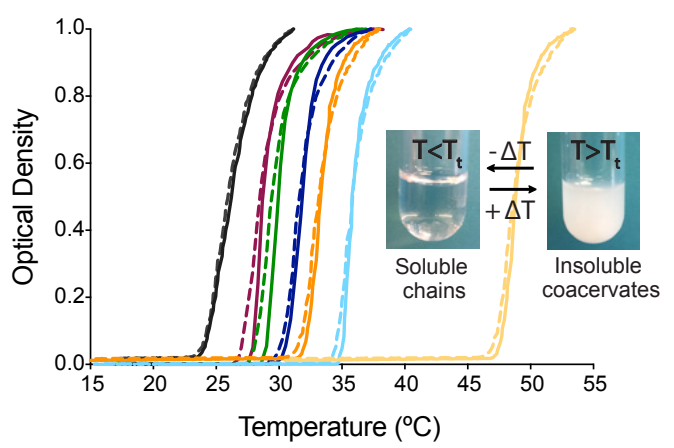

d

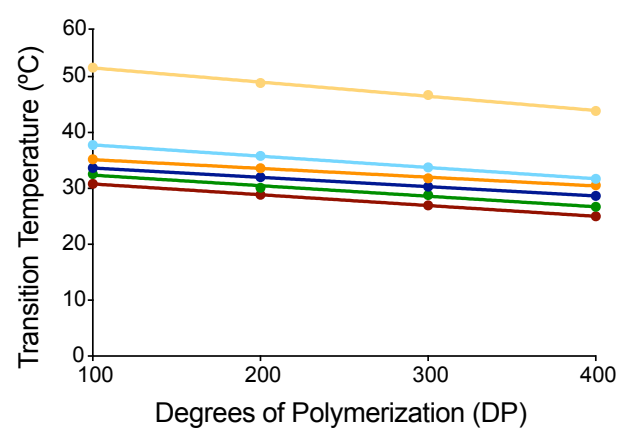

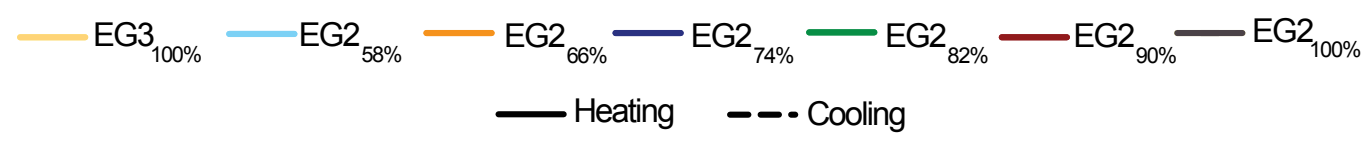

121 Figure 1. Well-defined POEGMAs of varying $\boldsymbol{M}_{\mathrm{w}}$ and monomer composition phase-transition near body temperature in a concentration-

122 and $\boldsymbol{M}_{\mathrm{w}}$-dependent manner and remain soluble at room temperature. (A) GPC chromatogram of EG2 $2_{66 \%}$ copolymers at varied $M_{w}$ (B) The 123 optical density of POEGMAs with constant DP but varying monomer composition was monitored as temperature increased (solid line) and 124 decreased (dashed line) to demonstrate the reversibility of phase behavior. Data were shown for POEGMAs at DP 200 and $25 \mu \mathrm{M}$. (C) At 125 varying concentrations, the optical density of POEGMAs demonstrates concentration dependence of $\mathrm{T}_{\mathrm{t}}$. Data were shown for POEGMAs at 126 DP 300 in phosphate-buffered saline (PBS). (D) The optical density of POEGMAs with varying DP and monomer composition was monitored 127 to demonstrate $T_{t}^{\prime}$ 's DP dependence. Data were shown for POEGMAs at $25 \mu \mathrm{M}$ in PBS.

128 Design and purification of a site-specific 1:1 conjugate. Conventional conjugation methods typically provide 129 limited control over the conjugation site and stoichiometry, resulting in a heterogeneous mixture of conjugates 130 with non-uniform PK and pharmacodynamics (PD). ${ }^{29}$ To circumvent this issue, we site-specifically conjugated 131 POEGMA to exendin at its C-terminus using bio-orthogonal click chemistry ${ }^{18,29-31}$ We chose the C-terminus as the 
132 POEGMA conjugation site, as the $\mathrm{N}$-terminus of exendin is critical to its function. ${ }^{18}$ To do so, we first covalently 133 conjugated a bio-orthogonal triglycine dibenzocyclooctyne (DBCO) group to the C-terminus of exendin via sortase 134 A-mediated native peptide ligation, ${ }^{18,30}$ yielding exendin-DBCO. To ensure that the conjugation is both 135 bioorthogonal -to avoid side-reactions of POEGMA with any internal residue in exendin - and site-specific, we 136 chose to synthesize azide-terminated POEGMA copolymers. The azide moiety readily reacts with the DBCO group 137 and does not react with any other chemical groups in exendin, yielding a site-specific conjugate with a 1:1 138 stoichiometry. ${ }^{30}$ (Supplementary Fig. 11).

Purification of POEGMA conjugates from unreacted agents using chromatography techniques is labor140 intensive and has a low yield. To circumvent these problems, we exploited the LCST behavior of depot-forming 141 POEGMA conjugates. The conjugates were purified in a chromatography-free manner at room temperature by 142 simply triggering the phase transition of the conjugate by adding ammonium sulfate salt that lowers the $T_{t}$ below 143 room temperature, ${ }^{32}$ leading to phase separation of Ex-POEGMA conjugates into an insoluble coacervate. 144 Unreacted exendin remained in the supernatant. The Ex-POEGMA coacervates were isolated by centrifugation 145 and were then resolubilized by adding PBS at room temperature. Importantly, this purification technique does not 146 apply to PEG as it does not show an LCST phase behavior.

147 Identifying an optimal Ex-POEGMA conjugate. We identified an optimal Ex-POEGMA conjugate that maximized 148 fed blood glucose control in hyperglycemic mice by first optimizing the $T_{t}$, then the $M_{w}$, and finally the dose of the 149 conjugate. POEGMA copolymers were synthesized for the $T_{t}$ optimization study are shown in Table 1 under the 150 heading -Index: $T_{t}$ and the copolymers with varied $M_{w}$ that were synthesized for the $M_{w}$ optimization study are 151 listed under the heading -Index: $M_{w}$.

152 Table 1. Characterization of exendin variants. EG2\% was calculated using NMR spectroscopy. $M_{n}, M_{w}$, and $Ð$ values were determined by 153 size exclusion chromatography multi-angle light scattering (SEC-MALS). $R_{h}$ was measured by dynamic light scattering (DLS) ( $\left.n=10\right)$. DP was 154 calculated by dividing polymer $M_{w}$ by the monomer $M_{w}$. The $E C_{50}$ of exendin variants was determined from the dose-response curves in 155 Fig. 2c, Fig. 2i, and Fig. 3c ( $n=10)$. Data are reported as mean \pm standard error of the mean (SEM). $T_{t}$ was measured at $500 \mu M$. *Calculated 
from the amino acid sequence. +Default value due to the monodisperse nature of the peptide. The indices in column 1 of the table $-T_{t}$,

$157 M_{w}$, and polymer type are explained in the text.

\begin{tabular}{|c|c|c|c|c|c|c|c|}
\hline Compound & EG2 \% & DP & $\mathrm{T}_{\mathrm{t}}\left({ }^{\circ} \mathrm{C}\right)$ & $M_{w}(\mathrm{kDa})$ & $\theta\left(M_{w} / M_{n}\right)$ & $R_{h}(n m)$ & $\mathrm{EC}_{50}(\mathrm{nM})$ \\
\hline Exendin & N/A & N/A & N/A & $4.2^{*}$ & $1.00^{+}$ & $2.2 \pm 0.1$ & $0.04 \pm 0.01$ \\
\hline \multicolumn{8}{|l|}{ Index: $T_{t}$} \\
\hline Ex-POEGMA $A_{31.99^{\circ} \mathrm{C}}$ & 48.5 & 247 & 31.9 & 57.7 & 1.15 & $4.8 \pm 0.7$ & $4.7 \pm 0.8$ \\
\hline Ex-POEGMA $29.99^{\circ} \mathrm{C}$ & 60.5 & 253 & 29.9 & 57.6 & 1.15 & $4.4 \pm 0.9$ & $3.0 \pm 0.7$ \\
\hline Ex-POEGMA $28.4^{\circ} \mathrm{C}$ & 68.8 & 254 & 28.4 & 56.9 & 1.13 & $4.5 \pm 0.7$ & $4.5 \pm 1.1$ \\
\hline \multicolumn{8}{|l|}{ Index: $M_{w}$} \\
\hline Ex-POEGMA $18.9 \mathrm{kDa}$ & 100 & 71 & 32.3 & 18.9 & 1.03 & $2.6 \pm 0.9$ & $1.2 \pm 0.4$ \\
\hline Ex-POEGMA ${ }_{54.3 \mathrm{kDa}}$ & 62.5 & 239 & 29.6 & 54.3 & 1.14 & $4.2 \pm 0.5$ & $2.8 \pm 0.7$ \\
\hline Ex-POEGMA & 56.8 & 454 & 29.0 & 99.4 & 1.13 & $4.5 \pm 1.1$ & $1.4 \pm 0.3$ \\
\hline Ex-POEGMA ${ }_{171.4 k D a}$ & 50.8 & 792 & 29.4 & 171.4 & 1.16 & $5.1 \pm 1.0$ & $1.8 \pm 0.2$ \\
\hline \multicolumn{8}{|l|}{ Index: Polymer type } \\
\hline Ex-POEGMA ${ }_{\text {opt }}$ & 62.3 & 241 & 29.9 & 54.7 & 1.09 & $4.1 \pm 0.5$ & $2.8 \pm 0.7$ \\
\hline Ex-POEGMA ${ }_{\text {sol }}$ & N/A & 221 & 40.1 & 56.6 & 1.09 & $4.3 \pm 0.3$ & $3.6 \pm 0.9$ \\
\hline Ex-PEG ${ }_{R h}$ & N/A & 454 & N/A & 27.7 & 1.07 & $4.4 \pm 1.1$ & $1.3 \pm 0.2$ \\
\hline$E x-P E G_{M w}$ & N/A & 909 & N/A & 46.3 & 1.10 & $5.2 \pm 1.0$ & $2.7 \pm 0.5$ \\
\hline
\end{tabular}

In the first phase of optimization, to identify an Ex-POEGMA conjugate with an optimal $T_{t}$, we first conjugated exendin to POEGMAs that have a varied monomer composition and hence $T_{t}$, but all of which had a near-constant $M_{w}$ of $\sim 57 \mathrm{kDa}$ (Table 1; index $\mathrm{T}_{\mathrm{t}}$ ). We chose an $M_{w}$ of $\sim 57 \mathrm{kDa}$, as it was previously shown to be

162 the $M_{\mathrm{w}}$ that maximized the PK of a soluble Ex-POEGMA conjugate (Table 1; Supplementary Fig. 12). ${ }^{18}$ All 163 conjugates had a low polydispersity and had a similar $R_{h}$ (Table 1; Supplementary Fig. 13). In addition, they showed reversible phase behavior (Fig. $2 \mathrm{a}$ ), and the $\mathrm{T}_{\mathrm{t}}$ of all conjugates were between $28-32^{\circ} \mathrm{C}$, allowing the conjugates to remain in solution at room temperature and transition into an insoluble coacervate when injected s.c. at an 166 injection concentration of $500 \mu \mathrm{M}$ (see circled data in Fig. 2b). The $T_{t}$ of the conjugates also showed an inverse concentration-dependence (Fig. 2b), indicating that they should release from the depot into the blood in response to dilution at the depot's margins. The conjugates with a greater EG2 content had a lower $T_{t}$, as the EG2 unit is 169 more hydrophobic than the EG3 unit. (Table 1; Supplementary Fig. 14). Finally, the conjugates were tested for 170 their ability to activate exendin's endogenous receptor, termed glucagon-like peptide 1 receptor (GLP1R), in an in 171 vitro cell-based assay using unconjugated exendin and PBS as controls. The conjugates showed high potency in 
172 activating GLP1R (Fig. 2c) but had a lower half-maximal effective concentration $\left(E C_{50}\right)$ than exendin due to the

173 steric hindrance imparted by the conjugated POEGMA, consistent with the literature. ${ }^{18,25,30}$ No significant 174 difference was observed in the $\mathrm{EC}_{50}$ of depot-forming conjugates.
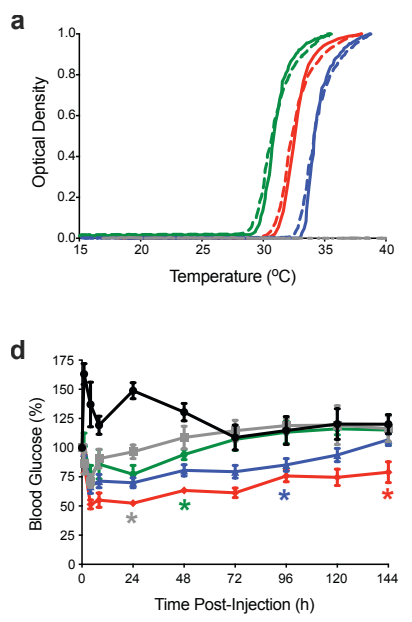

g

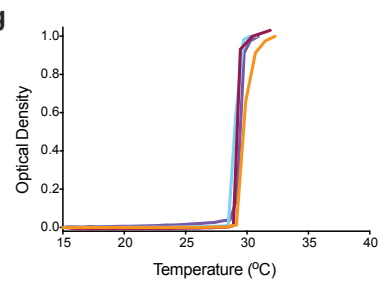

j

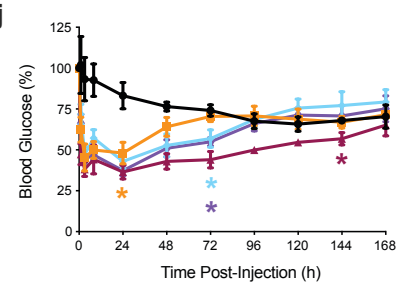

b

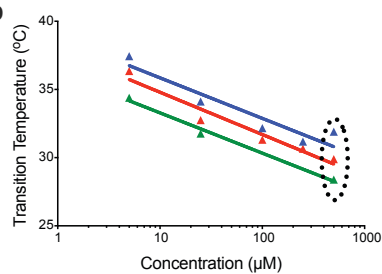

e
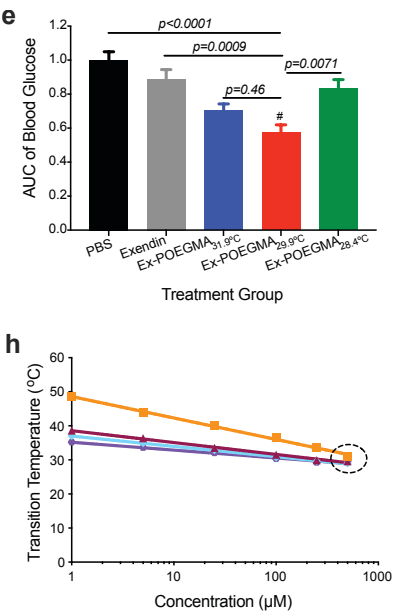

k
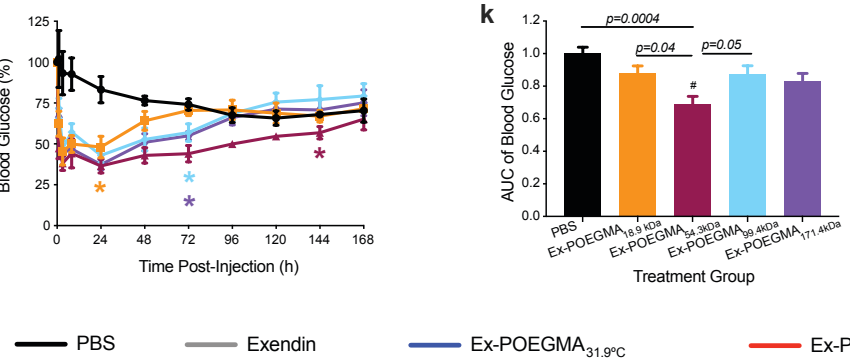

Ex-POEGMA ${ }_{18.9 k \mathrm{ka}} \quad$ Ex-POEGMA $_{54.3 \mathrm{kDa}}$
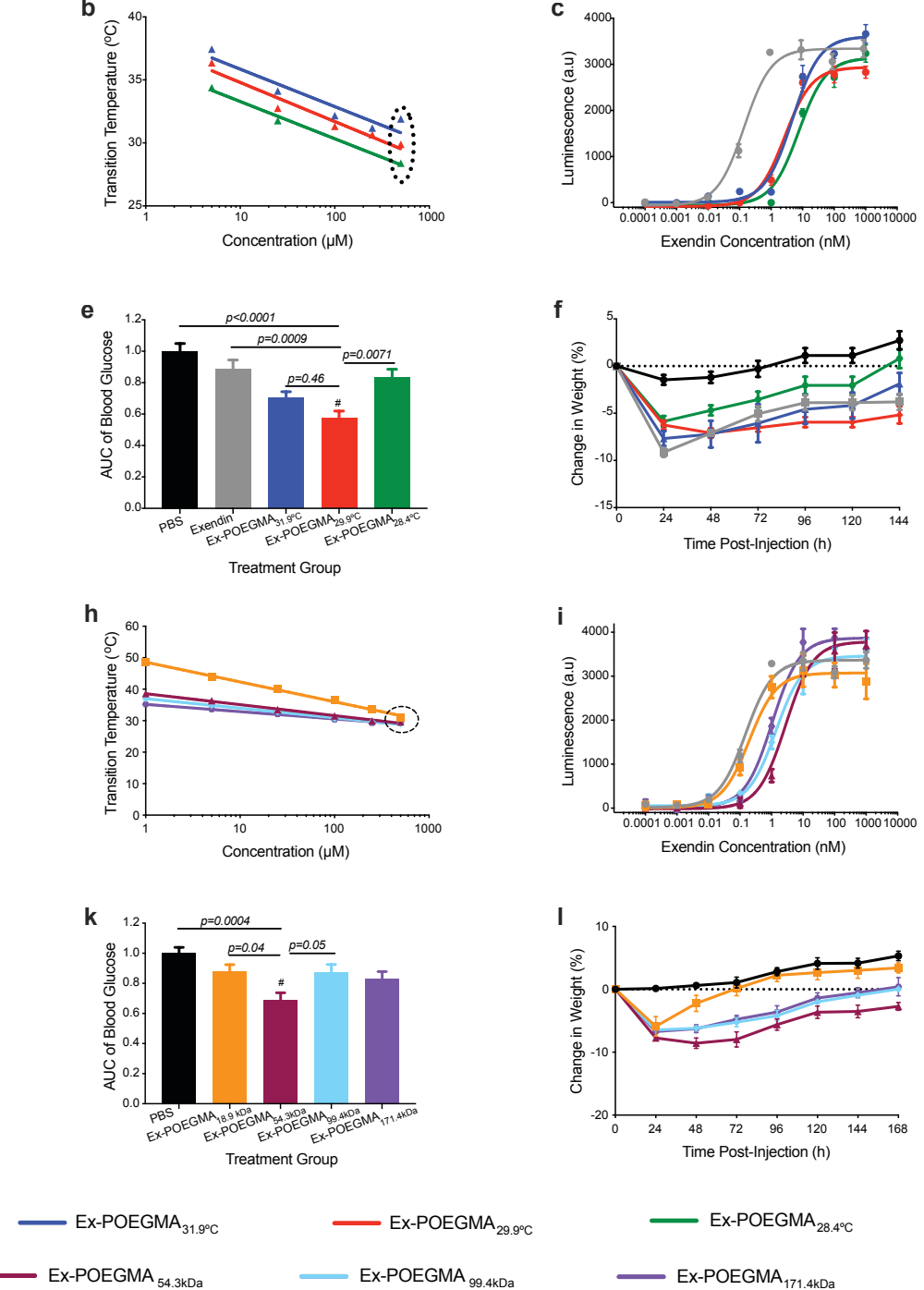

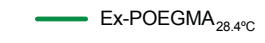

EX-POEGMA

Figure 2. Identifying an optimal Ex-POEGMA conjugate that maximizes fed blood glucose control in hyperglycemic mice. (A) The optical density of Ex-POEGMA conjugates with similar $M_{w}$ but varied $T_{t}$ as temperature increased (solid line) and decreased (dashed line), demonstrating the reversibility of phase behavior at $25 \mu \mathrm{M}$ and (B) at varying concentrations to demonstrate inverse concentration-

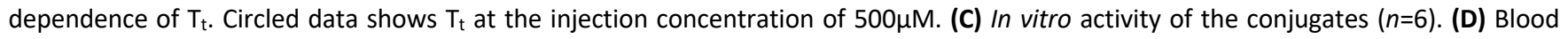
glucose normalized to $\mathrm{t}=0$, (E) AUC of blood glucose, and (F) percent weight change relative to weight at $t=0$ after treating $\mathrm{DIO} C 57 \mathrm{BL} / 6 \mathrm{~J}$ mice $(n=6)$ with a single s.c. injection of the treatments. (G) The optical density of Ex-POEGMA conjugates with optimal $T_{t}$ but varied $M_{w}$ as 
temperature increased, demonstrating phase behavior at $25 \mu \mathrm{M}$ and $(\mathbf{H})$ at varying concentrations to demonstrate inverse concentrationdependence of $\mathrm{T}_{\mathrm{t}}$. (I) In vitro activity of the conjugates ( $\left.n=6\right)$. (J) Blood glucose normalized to $\mathrm{t}=0$, (K) AUC of blood glucose, and (L) percent weight change relative to weight at $t=0$ after treating DIO C57BL/6J mice $(n=6)$ with a single s.c. injection of the treatments. Data were analyzed by two-way repeated-measures ANOVA followed by post hoc Dunnett's multiple comparison test. *The last time point that blood glucose for treatment is significantly lower than that of PBS treated mice. The AUC for glucose exposure was analyzed using two-way ANOVA, followed by post-hoc Tukey's multiple comparison test. \#The conjugate with the lowest AUC for glucose exposure. Data showed the mean \pm SEM and were considered statistically significant when $p<0.05$.

We next examined the fed blood glucose control provided by the Ex-POEGMA conjugates by administering them s.c. into 11-week-old male diet-induced obese (DIO) ${ }^{33} \mathrm{C} 57 \mathrm{BL} / 6 \mathrm{~J}$ mice. The equivalent dose of exendin and equivalent volume of PBS were used as positive and negative controls, respectively. Mice treated with exendin and the conjugates had lower blood glucose levels (Fig. 2d; Supplementary Fig. 15) and body weight (Fig. 2f) than the PBS-treated group. Exendin provided a shorter temporal duration of control than the conjugates, likely due to its short half-life. ${ }^{34}$ The most hydrophobic conjugate tested-Ex-POEGMA 28.4 -provided only modest control, possibly because it released only a small amount of drug from the depot. The Ex-POEGMA 29.9 conjugate outperformed others by providing six days of glucose control and had the lowest area under the curve (AUC) for glucose exposure (Fig. 2e), which accounts for both magnitude and duration of blood glucose reduction. This result was consistent with the literature, where the optimal $T_{t}$ of an injectable elastin-like polypeptide (ELP) drug depot was also reported to be $\sim 30$ ㅇ․ ${ }^{25}$

Having identified an Ex-POEGMA conjugate with an optimal $T_{t}$, we next identified an optimal $M_{w}$. Our strategy was to synthesize Ex-POEGMA conjugates with the optimal $\mathrm{T}_{t}$ of $\sim 30^{\circ} \mathrm{C}$ but with varying $M_{w}$ (Table 1; Index $\boldsymbol{M}_{\boldsymbol{w}}$ )-18.9 kDa, $54.3 \mathrm{kDa}, 99.3 \mathrm{kDa}$, and $171.4 \mathrm{kDa}$ (Supplementary Fig. 16). As the $T_{t}$ is a function of the $M_{w}$, we carried out an iterative set of polymer syntheses to create POEGMA copolymers that have the similar $T_{t}-$ close

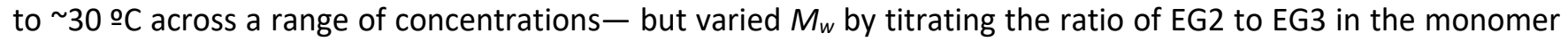
feed of the synthesis, leading to the set of polymers shown in Table 1 and listed under Index: $M_{w}$. The polymers with a lower $M_{w}$ had a greater mole fraction of the more hydrophobic EG2 monomer in the POEGMA copolymers 
and vice versa (Supplementary Fig. 17). These polymers were then conjugated to exendin. The resulting Ex-

208 POEGMA conjugates had varied $R_{h}$ (Supplementary Fig. 18) consistent with their different $M_{w}$, but all showed 209 thermally reversible phase behavior (Fig. 2g). However, the $T_{t}$ of the conjugates had a slightly different inverse 210 concentration dependence, as seen by the different slopes of the log(concentration) versus $\mathrm{T}_{\mathrm{t}}$ plots (Fig. 2h). 211 Importantly, these plots intersected at $500 \mu \mathrm{M}$ and $\sim 30^{\circ} \mathrm{C}$ (see circled data in Fig. $2 \mathrm{~h}$ ), a temperature that 212 fortuitously corresponds to the previously identified optimal $T_{t}$. This concentration of $500 \mu \mathrm{M}$ was hence chosen 213 as the injection concentration for all in vivo studies to identify the optimal $M_{w}$ with constant $T_{t}$ of $\sim 30$ 으. No 214 significant correlation was observed between in vitro activity of the conjugates as seen by their $\mathrm{EC}_{50}$ and the $M_{w}$ 215 of conjugates (Fig. 2i).

The conjugates were next administrated into 11-week-old male DIO mice $(n=6)$, with PBS as a negative control. Mice treated with the conjugates had lower blood glucose (Fig. 2j; Supplementary Fig. 19) and body weight (Fig. 2l) than the PBS-treated group. The conjugate with the lowest $M_{w}$ tested-Ex-POEGMA $18.9-$ controlled blood glucose only one day and had a significantly higher glucose exposure as seen by the AUC (Fig. 2k) than all other treatment groups. This data suggests that Ex-POEGMA $18.9 \mathrm{kDa}$ was cleared from the circulation much faster than the other conjugates because of its significantly smaller size $\left(R_{h} \sim 2.6\right.$ vs. $\sim 4-5 \mathrm{~nm}$ for the other conjugates) that is lower than the renal excretion threshold, defined as the size of serum albumin $(\sim 3.6 \mathrm{~nm}){ }^{18,25}$ This data is consistent with the literature, where PEG and ELP conjugates show a prolonged half-life at an $M_{w}$ threshold of $30 \mathrm{kDa}^{35,36}$ and $35.8 \mathrm{kDa}^{25}$, respectively. In addition, the higher $M_{w}$ Ex-POEGMA conjugates differed in their duration of glucose control. Ex-POEGMA ${ }_{99.4}$ and Ex-POEGMA ${ }_{171.4}$ showed modest glucose control for three days, while Ex-POEGMA ${ }_{54.3}$ outperformed the rest with six days of glucose control and had the lowest AUC for glucose exposure. This conjugate - Ex-POEGMA ${ }_{54.3}-$ was defined as the $T_{t^{-}}$and $M_{w^{-}}$optimized conjugate and is subsequently referred to as Ex-POEGMA opt in this paper. Finally, the optimal injection dose of this conjugate was determined to be $1000 \mathrm{nmol} \mathrm{kg}^{-1}$ in a dose-escalation study, which completed the optimization process of the conjugate (Supplementary Fig. 20). 
231 Short-term efficacy. We next investigated the short-term efficacy of Ex-POEGMA ${ }_{\text {opt }}$ compared to its soluble 232 POEGMA and PEG counterparts. We synthesized the soluble counterpart of Ex-POEGMA opt -termed Ex-

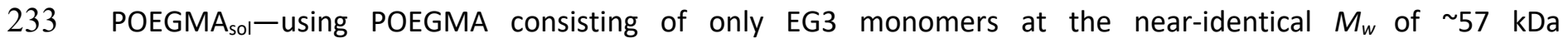
234 (Supplementary Fig. 21). In preliminary studies, we found that linear PEG has a much larger hydrodynamic size 235 than hyperbranched POEGMA of the same $M_{w}$, resulting in conjugates with a much larger $R_{h}$ at the same $M_{w}$. 236 Because this difference could complicate side-by-side efficacy comparison of the conjugates by affecting their 237 kidney clearance rates, we synthesized both $M_{w}$-matched and $R_{h}$-matched exendin-PEG conjugates-termed Ex-

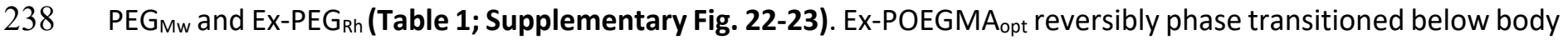
239 temperature (Fig. 3a), allowing it to remain as a solution in a syringe at room temperature but to transition into 240 an insoluble coacervate phase when injected s.c., as tested at a concentration of $500 \mu \mathrm{M}$ (see circled data in Fig. 2413 3b). In contrast, Ex-POEGMA ${ }_{\text {sol }}$ phase-transitioned well above body temperature at all concentrations, indicating 242 that it cannot form a depot and would remain soluble when injected into the s.c. space. Neither Ex-PEG $\mathrm{Mw}_{w}$ nor Ex$243 \mathrm{PEG}_{\mathrm{Rh}}$ showed phase behavior (Fig. 3a). The conjugates showed no difference in their $\mathrm{EC}_{50}$ in activating the GLP1R 244 in an in vitro, cell-based assay (Fig. 3c).

The conjugates were next administered s.c. into 11-week-old male DIO mice $(n=6)$ at the equivalent, 246 optimal dose, with PBS included as a control. Mice treated with the conjugates had lower fed blood glucose levels 247 than the control (Fig. 3d; Supplementary Fig. 24). Ex-PEG $G_{R h}$ and Ex-PEG ${ }_{M w}$ controlled fed blood glucose for three 248 and four days, respectively (Fig. 3d), consistent with the literature. ${ }^{37}$ The one-day longer glucose control provided 249 by Ex-PEG ${ }_{M w}$ could be attributed to its larger size, and hence slower clearance. In accord with the literature, Ex250 POEGMA sol $_{\text {also provided four days of blood glucose control. }{ }^{18} \text { In contrast, Ex-POEGMA }}$ opt outperformed its soluble 251 POEGMA counterpart and the two PEGylated exendin controls by providing six days of fed blood glucose control 252 and had the lowest glucose exposure of all conjugates (Fig. 3e), showing the enhanced efficacy provided by 253 sustained release of the conjugate from an injectable s.c. depot. All soluble conjugates resulted in a much sharper 254 and more significant weight loss than Ex-POEGMA opt (Fig. 3f), possibly because a sudden increase in drug 
concentration of soluble conjugates can induce nausea and trigger transient weight loss, which is consistent with 256 common side effects of exendin. ${ }^{38}$

model-the $d b / d b$ line - that displays more severe hyperglycemia than DIO mice. $d b / d b$ mice carry mutant leptin receptor gene and have a progressive course of declining insulin secretion and hyperglycemia, similar to many 260 humans with T2D. ${ }^{33}$ In this study, we also compared Ex-POEGMA opt $_{\text {with }}$ Bydureon, a once-weekly FDA-approved 261 and clinically used sustained-release formulation of exendin encapsulated in poly-lactic-co-glycolic acid (PLGA) 262 microspheres. ${ }^{39}$ All treatment groups $(n=5)$ resulted in lower glucose levels than the PBS control (Fig. 3g; 263 Supplementary Fig. 25). Ex-POEGMA ${ }_{\text {opt, }}$ however, stood out from the competition by lowering fed blood glucose 264 for six days. A clear trend in glucose exposure (Fig. 3h) was also observed, with Ex-POEGMA opt $_{\text {having the lowest }}$ AUC. Soluble Ex-PEG ${ }_{R h}$ induced a more significant weight loss than sustained-release formulations Ex-POEGMA $A_{o p t}$ and Bydureon (Fig. 3i), consistent with previous results. an intraperitoneal (i.p.) glucose tolerance test (IPGTT) in $d b / d b$ mice on days 1,3 , and 5 post-injection of the treatments. Exendin-treated mice displayed similar glucose intolerance compared to the PBS-treated mice, indicated by hyperglycemia, presumably because it cleared from circulation before IPGTTs were performed due to its short half-life (Fig. 3j-I). ${ }^{34,40}$ There was a clear trend in glucose exposure on days 1 and 3 (Supplementary

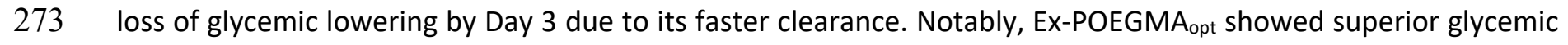
274 regulation to Ex-PEG ${ }_{\mathrm{Rh}}$ throughout the study by providing the lowest glucose exposure among the treatments. This 275 difference was also observed when the experiment was repeated using DIO mice (Supplementary Fig. 27). 276 However, the magnitude of the difference was not as great, possibly due to the milder display of T2D allowing 277 lower circulating Ex-PEG ${ }_{R h}$ concentrations to show therapeutic effect. The test time did not affect glucose 

superior glycemic regulation ability throughout the study.

a
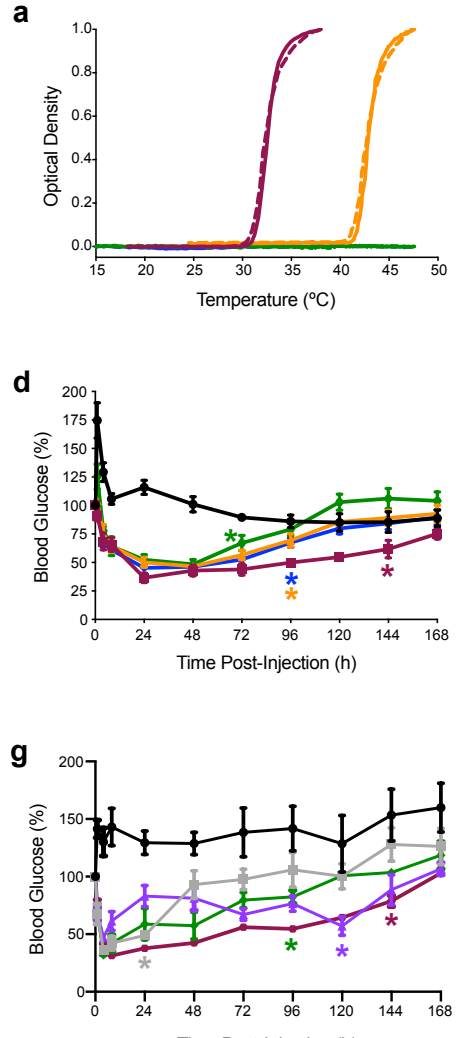

Time Post-Injection (h)
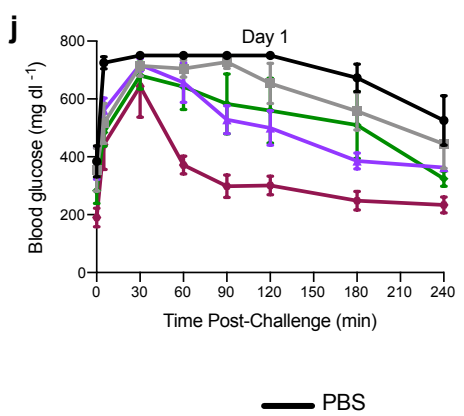

b

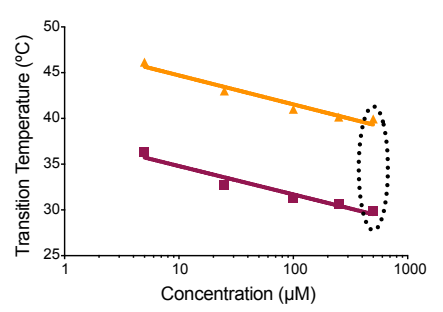

e

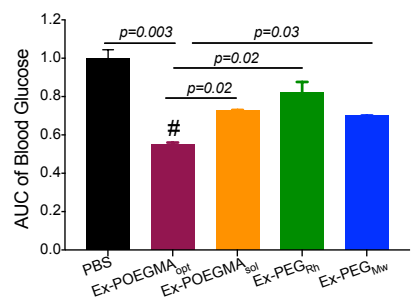

Treatment Group

h

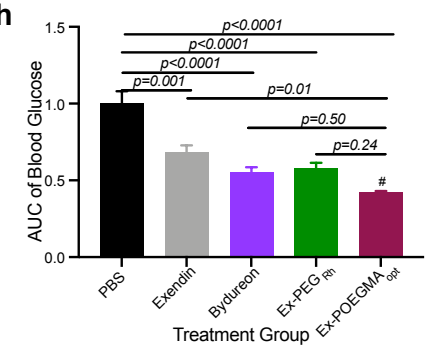

$\mathbf{k}$

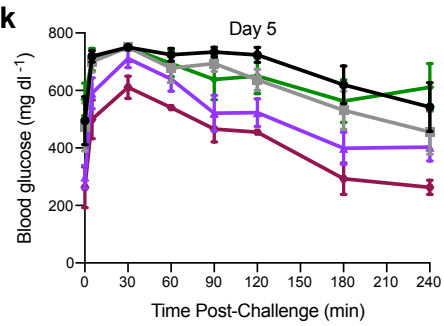

$\longrightarrow$ Ex$_{-P E G_{M w}}$

Exendin

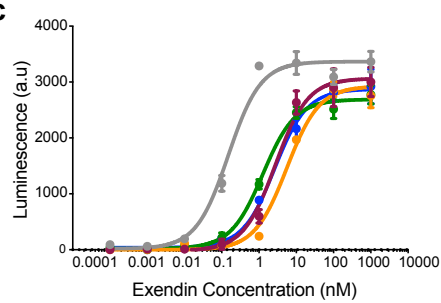

f

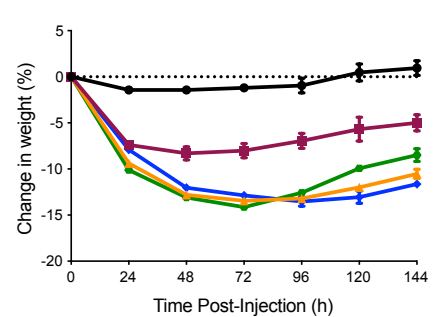

i

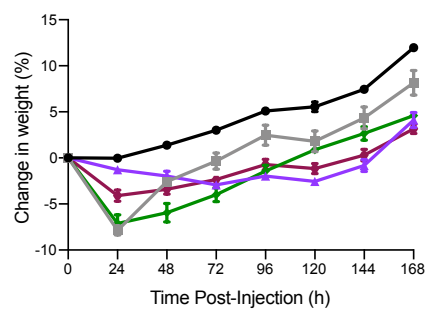

I

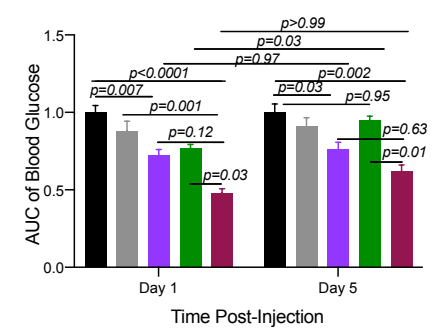

Ex-POEGMA

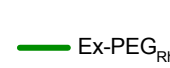

Ex-POEGMA

Bydureon

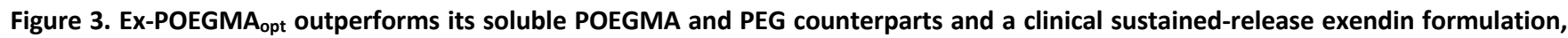

Bydureon, in fed blood glucose and glycemic control. The optical density of treatments (A) as temperature increased and decreased,

demonstrating reversible phase behavior for Ex-POEGMA $\mathrm{opt}_{\text {t }}$ and Ex-POEGMA $\mathrm{Aol}_{\text {al }}$ at $25 \mu \mathrm{M}$ and (B) at varying concentrations to demonstrate 
DIO mice $(n=6)$ with a single s.c. injection of the treatments. (G) Blood glucose normalized to $t=0,(\mathbf{H})$ AUC of blood glucose, $(\mathbf{F})$ and percent weight change relative to weight at $t=0$ after treating six-week-old $d b / d b$ mice $(n=6)$ with a single s.c. injection of the treatments. An IPGTT was performed on ( $\mathrm{J})$ day 1 and (K) day 5 post-injection of treatments into $d b / d b$ mice $(n=5)$, blood glucose was monitored, and (L) AUC of blood glucose was quantified. Data were analyzed by two-way repeated-measures ANOVA, followed by post hoc Dunnett's multiple comparison test. *The last time point that blood glucose for treatment is significantly lower than that of PBS treated mice. The AUC for glucose exposure was analyzed using two-way ANOVA, followed by post-hoc Tukey's multiple comparison test. \#The conjugate with the lowest AUC for glucose exposure. Data showed the mean \pm SEM and were considered statistically significant when $p<0.05$.

Pharmacokinetics. We next investigated the PK of Ex-POEGMA ${ }_{\text {opt }}$ and the PEGylated controls to better understand the differences in the short-term efficacy profiles of the treatments. We fluorescently labeled exendin, Ex-

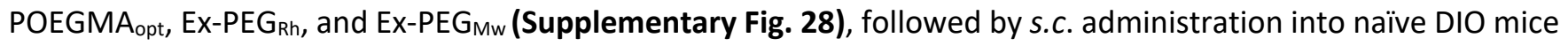
$(n=4)$. The PK parameters were determined from the drug's plasma concentration (Fig. 4a-b) and are shown in Table 2. All conjugates had much slower absorption kinetics than exendin, suggesting that the conjugates were primarily taken up from the s.c. space into the blood through the lymphatic system due to their large size. ${ }^{41}$ ExPOEGMA ${ }_{\text {opt }}$ had the slowest absorption ( $t_{1 / 2}$ absorption $=9.0 \pm 1.8 \mathrm{~h}$ ) among the treatments, possibly due to its amphiphilic structure and sustained release. Exendin had a fast elimination half-life $\left(\mathrm{t}_{1 / 2}\right.$ elimination $\left.=2.1 \pm 0.5 \mathrm{~h}\right)$, defined as the time needed to reach half-maximal blood concentration, consistent with its small size (Fig. 4a). ${ }^{40}$ The conjugates had much longer elimination half-lives due to their slower renal clearance (Fig. 4b). Ex-PEG $\mathrm{Rh}_{1 / 2}$ elimination $=23.1 \pm 6.4 \mathrm{~h})$ and $E x-P E G_{M w}\left(t_{1 / 2}\right.$ elimination $\left.=34.9 \pm 9.1 \mathrm{~h}\right)$ prolonged exendin's circulation by $\sim 11$ - and $\sim 17$-fold, respectively. The $55.6 \mathrm{kDa}$ soluble Ex-POEGMA conjugate with a matching $M_{w}$ as the optimal depot-forming

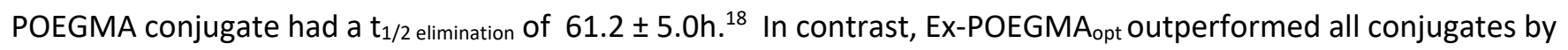
prolonging exendin's circulation by $\sim 46$-fold with a $t_{1 / 2}$ elimination of $97.3 \pm 5.6 \mathrm{~h}$, and it provided the highest drug exposure (AUC) among the treatments. These results clearly demonstrate the PK benefits of sustained release 


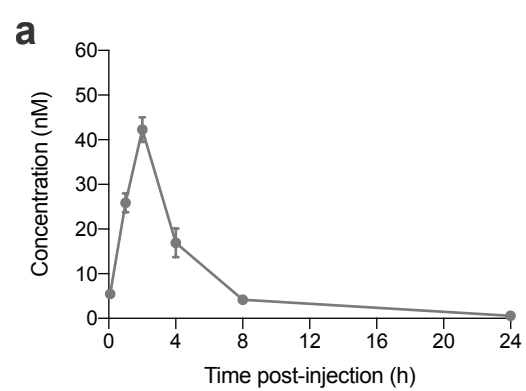

d

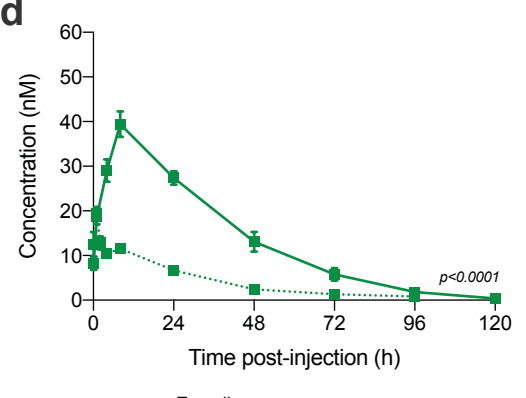

Exendin b

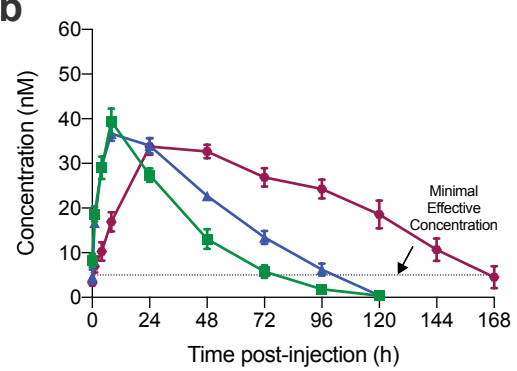

e
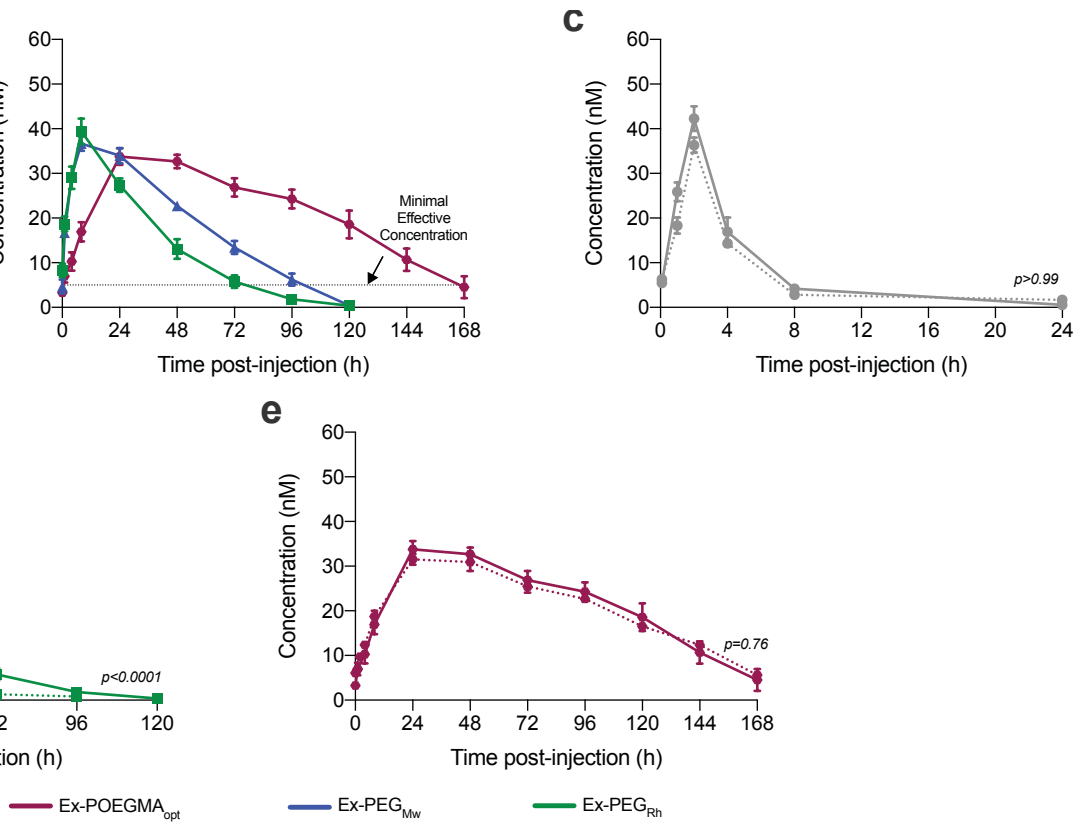

_ Naive mice
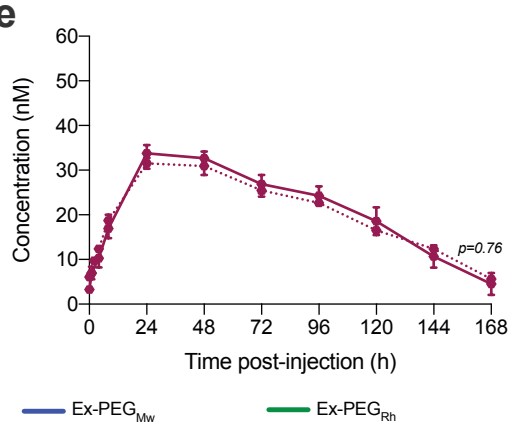

...... Immunized mice

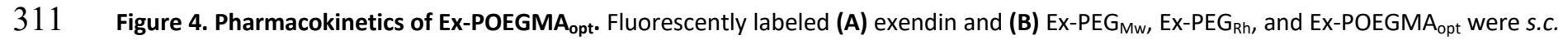
administrated into naïve male DIO C57BL/6J mice $(n=4)$ at $1000 \mathrm{nmol} \mathrm{kg}^{-1}$, followed by blood collection at specified time points for 168 hours to calculate drug concentration. The treatments were administrated four more times to immunize the mice and induce ADAs. At the last injection, concentrations of fluorescently labeled (C) exendin, (D) Ex-PEG $G_{R h}$, and (E) Ex-POEGMA opt were tracked (dotted lines). $P$ values were shown for AUC comparison between naïve (solid line) and immunized mice for each treatment. Fluorophore concentration was 45 $\mathrm{nmol} \mathrm{kg}{ }^{-1}$. Data represent the mean $\pm \mathrm{SEM}$.

Long-term efficacy. We next investigated the long-term efficacy of Ex-POEGMA $A_{o p t}$ to understand better how the

318 differences in PK and short-term efficacy translated into long-term management of T2D. We hypothesized that

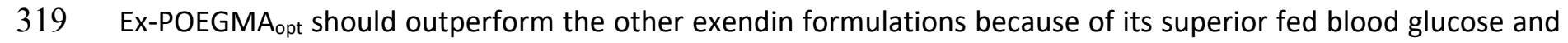

320 glycemic control and longer PK. We tested this hypothesis by administering sterile and endotoxin-free Ex321 POEGMA ${ }_{\text {opt }}, E_{x}-P_{\text {Ph }}$, Bydureon, exendin, and PBS (- control) s.c. into naïve six-week-old male $d b / d b$ mice $(n=5)$ 322 every week for eight weeks, followed by monitoring their blood glucose, changes in body weight, and glycated 323 hemoglobin ( $\mathrm{HbA} 1 \mathrm{c} \%$ ) levels. We chose to monitor $\mathrm{HbA} 1 \mathrm{c} \%$, as it is a measure of long-term T2D management 324 because it is insensitive to daily blood glucose fluctuations and only changes as red blood cells (RBC) turn over every 40-60 days in rodents. ${ }^{25}$ All treatments resulted in lower fed blood glucose levels (Fig. 5a; Supplementary 
326 Fig. 29) and glucose exposure (Fig. 5b) than the PBS control. Exendin only had a modest and short-lived effect due 327 to its poor circulation. Long-term treatment with Bydureon resulted in glycemic improvement (Fig. 5a-b). Ex-PEG 328 controlled fed blood glucose at varying degrees, with later injections providing less glycemic reduction, and 329 resulted in a $\mathrm{HbA} 1 \mathrm{c} \%$ level that was not significantly different from PBS-treated mice $(p>0.05)$. In contrast to the 330 other treatments, Ex-POEGMA ${ }_{\text {opt }}$ consistently lowered fed blood glucose control after each injection and provided 331 the lowest glucose exposure among all treatments. This profile was reflected by a lower HbA1c\% in Ex-POEGMA $\mathrm{opt}^{-}$ 332 treated mice than the levels attained with the other treatments. The sustained release formulations Bydureon

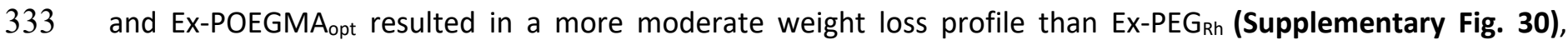
334 consistent with the results after a single injection of the treatments.

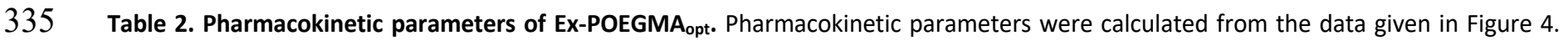
336 Data were fitted to a one-phase exponential decay curve and analyzed via non-compartmental pharmacokinetic analysis using GraphPad 337 Prism 8 software. Absorption phase pharmacokinetics of Ex-PEG $\mathrm{Rh}_{\mathrm{R}}$ in immunized mice were not calculated (N/C) due to its low goodness338 of-fit values. Data showed the mean \pm SEM.

\begin{tabular}{|c|c|c|c|c|c|c|}
\hline & Treatment & $t_{1 / 2 \text { absorption }}\left(h^{-1}\right)$ & $t_{1 / 2 \text { elimination }}\left(h^{-1}\right)$ & $t_{\max }(h)^{*}$ & $C_{\max }(n M) *$ & AUC ( $h \times n M)$ \\
\hline \multirow{4}{*}{$\begin{array}{l}\text { Naïve } \\
\text { Mice }\end{array}$} & Exendin & $0.6 \pm 0.1$ & $2.1 \pm 0.5$ & $1.6 \pm 0.2$ & $31.3 \pm 15$ & $188 \pm 18$ \\
\hline & $E x-P E G_{R h}$ & $6.6 \pm 0.5$ & $23.1 \pm 6.4$ & $16.6 \pm 2.2$ & $34.6 \pm 2.4$ & $1585 \pm 112$ \\
\hline & $E x-P E G_{M w}$ & $6.6 \pm 1.7$ & $34.9 \pm 9.1$ & $19.5 \pm 4.9$ & $35.3 \pm 4.5$ & $2206 \pm 84$ \\
\hline & Ex-POEGMA $A_{o p t}$ & $9.0 \pm 1.8$ & $97.3 \pm 5.6$ & $32.1 \pm 5.8$ & $35.8 \pm 2.6$ & $3664 \pm 167$ \\
\hline \multirow{3}{*}{$\begin{array}{l}\text { Immunized } \\
\text { Mice }\end{array}$} & Exendin & $0.8 \pm 0.1$ & $1.9 \pm 0.5$ & $1.7 \pm 0.3$ & $36.9 \pm 6.8$ & $159 \pm 18$ \\
\hline & $E x-P E G_{R h}$ & $\mathrm{~N} / \mathrm{C}$ & $11.4 \pm 5.4$ & $\mathrm{~N} / \mathrm{C}$ & $\mathrm{N} / \mathrm{C}$ & $424 \pm 35$ \\
\hline & Ex-POEGMA ${ }_{\text {opt }}$ & $12.1 \pm 2.0$ & $105.7 \pm 12$ & $42.6 \pm 6.4$ & $30.3 \pm 4.0$ & $3664 \pm 167$ \\
\hline
\end{tabular}




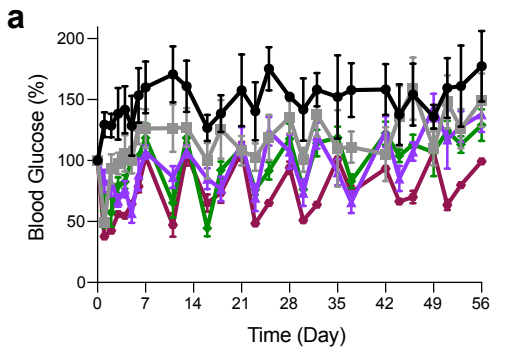

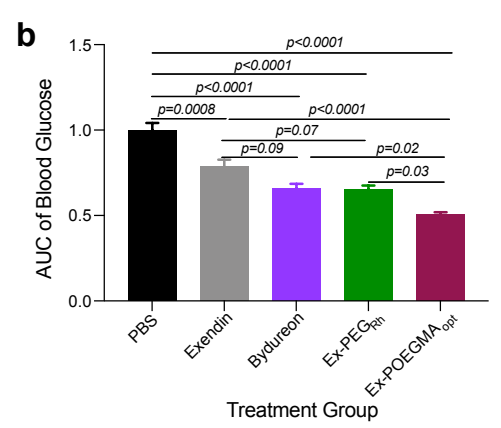
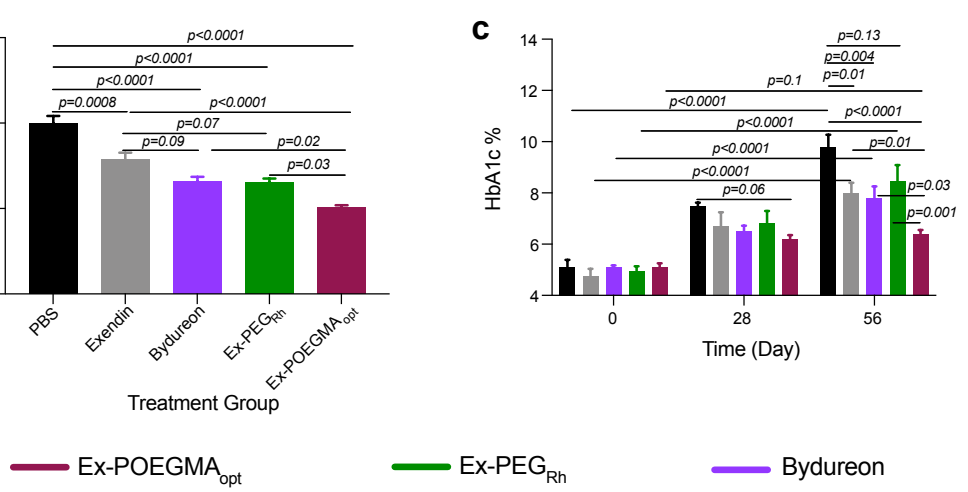

Bydureon

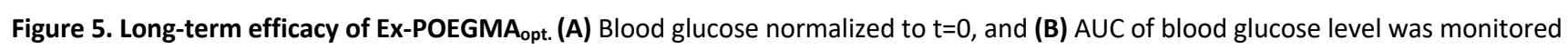
after treating $d b / d b(n=5)$ with weekly s.c. injections of exendin, Ex-PEG $G_{\mathrm{Rh}}$, Ex-POEGMA $\mathrm{Apt}_{\text {, }}$ Bydureon, or PBS for eight weeks. (C) Percent glycated hemoglobin (Hb1Ac\%). Data represent the mean \pm SEM. The AUC for glucose exposure and HbA1c\% were analyzed using twoway ANOVA, followed by post-hoc Tukey's multiple comparison test. Data were considered statistically significant when $p<0.05$.

Immunogenicity. We next tested the immunogenicity of POEGMA and compared it to PEG. PEG crosslinks the Bcell receptors (BCR) because of its linear repetitive structure, resulting primarily in a T-cell independent B-cell immune response. This response is characterized by a persistent and predominantly lgM response. ${ }^{42}$ In humans, IgG responses are also observed in some individuals, though the mechanism of class switching is not well understood. ${ }^{43}$ We hypothesized that POEGMA should not be immunogenic because of its hyperbranched structure and tested this hypothesis by assessing the induction of anti-drug antibodies (ADA) toward the full immunizing material (i.e., Ex-POEGMA ${ }_{\text {opt }}$ ). We administrated sterile and endotoxin-free Ex-POEGMA ${ }_{o p t}$, Ex-PEG $\mathrm{Mw}_{\text {, }}$ exendin, and PBS (- control) s.c. into naïve DIO mice $(n=10)$ (see dosing and blood sampling regimen in Fig. 6a) and assessed ADAs in terms of their titer, specificity (i.e., anti-conjugate, anti-exendin, anti-PEG, or anti-POEGMA), and subtype (i.e., IgM or $\lg G$ ) using a Luminex multiplexed immunoassay. Briefly, the Luminex immunoassay used drugconjugated, fluorescently barcoded magnetic beads to capture ADAs. We coupled exendin, Ex-PEG, and ExPOEGMA onto separate sets of beads to determine ADAs induced by the treatments. OVA versions of these beads-OVA, OVA-PEG, and OVA-POEGMA-were also coupled to separate sets of beads to use as a crossreactivity control and to determine PEG- and POEGMA-specific ADAs, respectively. We eliminated the possibility of detecting anti-linker antibodies by synthesizing OVA-PEG and OVA-POEGMA conjugates using a different 


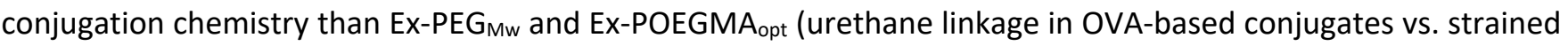

361 alkyne-azide linkage in exendin-based conjugates) (Supplementary Figure 31; Supplementary Table 2). Each drug-

362 conjugated bead set had a different fluorescent barcode, allowing us to measure the signal detected by each of

363 them when multiplexed. Mouse IgG- or IgM-coupled beads in diluent were used as positive controls, while the 364 multiplexed beads in diluent served as a negative control. The immunoassay's optimization and validation are 365 summarized in the Supplementary Information, and supplementary data are shown in Supplementary Fig. 31-32 and Supplementary Tables 2-8.

Ex-POEGMA $A_{\mathrm{opt}}$, Ex-PEG $\mathrm{Mw}_{\mathrm{w}}$ and exendin treatments induced a transient IgM response against exendin (Fig.

6b-c). No anti-exendin IgG response was detected (Supplementary Fig. 33). This data is consistent with the literature ${ }^{44}$, where only mild induction of anti-exendin antibodies was reported in clinical trials. In contrast, Ex$\mathrm{PEG}_{\mathrm{Mw}}$ induced a significant and persistent anti-PEG IgM response (Fig. 6b-c), consistent with the literature. ${ }^{7,8,42}$ 371 PEG specificity was indicated by the significant signal measured with both Ex-PEG and OVA-PEG beads. Anti-PEG 372 antibodies were strictly IgM, and no class switching to IgG was observed (Supplementary Fig. 33). PEG-specific

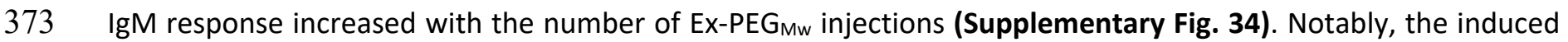
374 PEG antibodies did not bind POEGMA conjugated bead sets, indicating that POEGMA eliminates PEG antigenicity 375 in agreement with the literature. ${ }^{18,19}$ Strikingly, Ex-POEGMA ${ }_{\text {opt }}$ did not induce anti-POEGMA antibodies, indicating 376 that it is non-immunogenic.

We further tested the immunogenicity of POEGMA and compared it to PEG using highly immunogenic 378 OVA as its conjugation partner. Briefly, OVA, OVA-PEG, OVA-POEGMA, and PBS (negative control) were repeatedly 379 injected s.c. into naïve C57BL6/J mice $(n=10)$ in accordance with the timeline in Fig. 6a. The Luminex immunoassay 380 was used to quantify the ADAs induced by the treatments with minor changes from the previous experiment. 381 Briefly, OVA, OVA-PEG, and OVA-POEGMA coupled beads were used to quantify ADA responses to the full 382 immunizing material, while Ex-PEG and Ex-POEGMA coupled beads were employed as irrelevant protein 383 conjugates to measure responses to the polymer domains only. The exendin-coupled bead was used as a cross- 
384 reactivity control. Free, PEG-conjugated, and POEGMA-conjugated OVA induced significant anti-OVA IgG 385 responses by Day 44 (Supplementary Fig. 35a-b). The OVA-PEG conjugate induced lower titers of anti-OVA IgG 386 than unmodified OVA (Supplementary Fig. 35b). This result was attributed to the steric hindrance imparted by 387 the PEG, ${ }^{6}$ allowing the conjugate to expose fewer OVA epitopes to the immune system. The differences in anti388 OVA antibody titers were not as drastic when POEGMA was used. We speculate that this is because POEGMA 389 provides less steric hindrance than PEG to its conjugation partners due to its more compact architecture. ${ }^{45}$ PEG 390 was highly immunogenic, as seen by the high titers of PEG-specific ADAs detected in OVA-PEG-immunized mice 391 plasma with Ex-PEG beads (Supplementary Fig. 35c-d). Similar to when exendin was the conjugation partner, PEG392 specific ADAs were restricted to the IgM class (Supplementary Fig. 35c-d), possibly due to the lack of T-cell help 393 for class switching, as reported in the literature. ${ }^{42}$ The PEG-specific IgM titer was $\sim 60$-fold higher with OVA-PEG 394 than Ex-PEG, possibly due to the higher immunogenicity of OVA. In contrast, only a mild anti-POEGMA IgM 395 response was detected after the first drug injection. The anti-POEGMA response was not detectable at later time 396 points (Supplementary Fig. 36d) and did not mature into an IgG response (Supplementary Fig. 36h), further 397 supporting our conclusions that POEGMA appears to be significantly less immunogenic than PEG. 


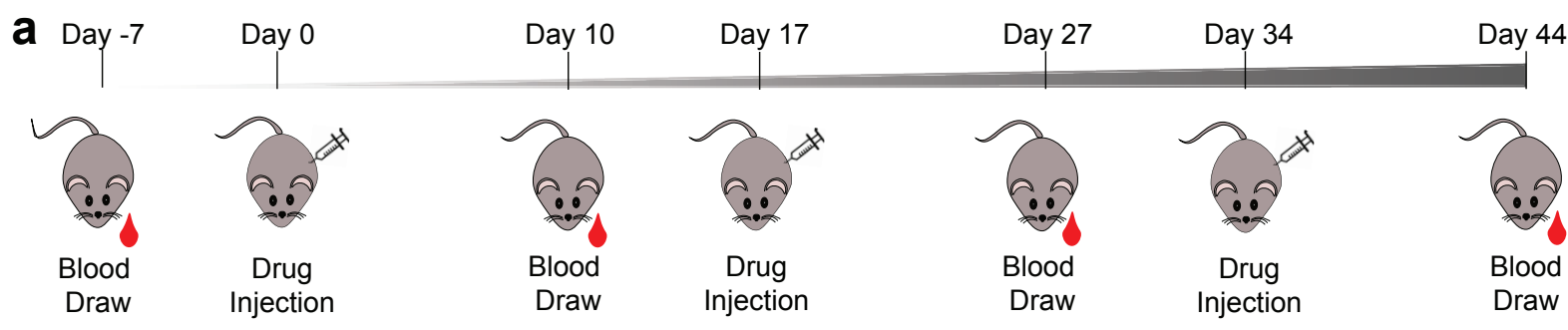

b

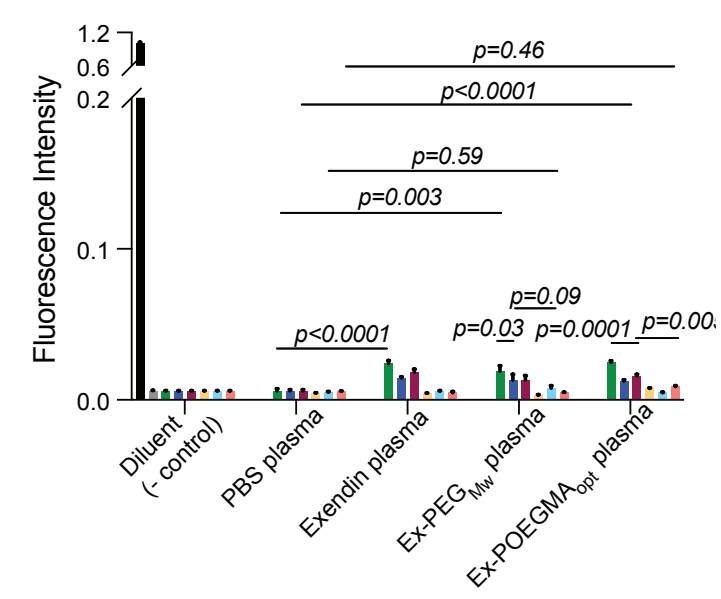

Test Group

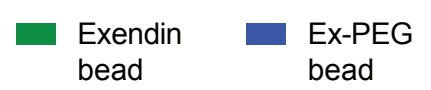

IgM Response-Day 10

C IgM Response-Day 44

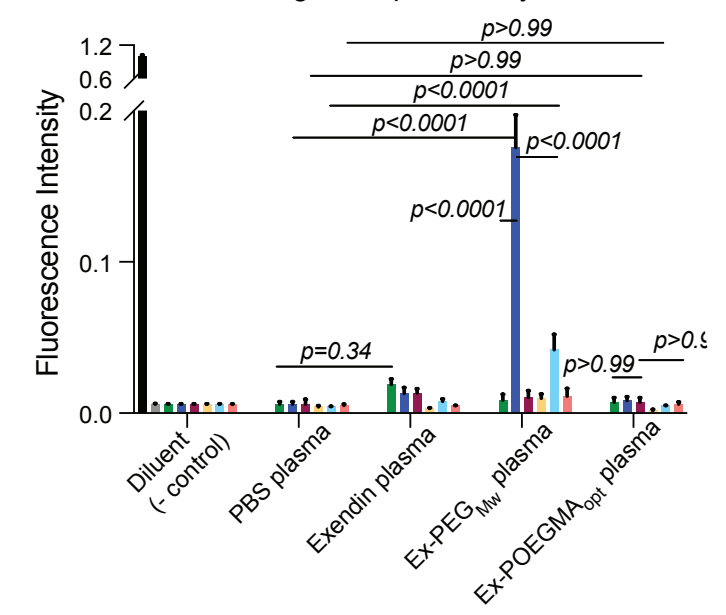

Test Group

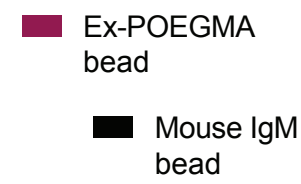

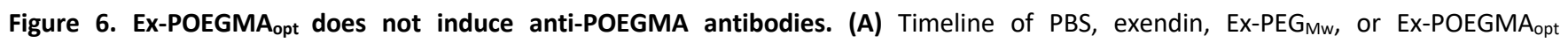

400 administration into DIO C57BL/6J mice ( $n=10)$ and blood collection. IgM subtype ADA response on (B) Day 10 and (C) Day 44 . Blood samples were collected from mice repeatedly treated with PBS, exendin, Ex-PEG $\mathrm{Mw}_{\mathrm{w}}$, or Ex-POEGMA $\mathrm{opt}_{\text {. }}$ ADA response was measured by a multiplexed Luminex immunoassay. Ex-PEG- and Ex-POEGMA-coupled beads were used to determine ADAs induced towards the entire conjugate (i.e., anti-exendin and anti-polymer - PEG or POEGMA). OVA-PEG- and OVA-POEGMA-coupled beads were used to determine ADAs induced towards PEG or POEGMA, respectively. The OVA-coupled beads were used as a negative control for cross-reactivity towards OVA. Data represent the mean ADA response induced in each mouse \pm SEM. Data were analyzed by two-way repeated-measures ANOVA, followed by post-hoc Tukey's multiple comparison test. Data were considered statistically significant when $p<0.05$. 
410 Freund's adjuvants (FA) significantly enhance the titer and affinity of the immune response induced towards co-

411 administered immunogens by extending their presentation, stimulating the innate immune system, and

412 facilitating T-cell help, commonly allowing immunogens to reveal their most immunogenic state. ${ }^{47}$ We

413 hypothesized that POEGMA should at most induce a weak IgM immune response. We tested this hypothesis by

414 administering PBS (- control), OVA, OVA-PEG, and OVA-POEGMA s.c. into naïve C57BL/6J mice ( $n=10)$ in FA

415 emulsions. Blood was collected by following the timeline in Fig. 6a, processed into plasma, and tested by a

416 Luminex immunoassay. Strikingly, a POEGMA-specific IgM response was observed transiently and disappeared

417 after the first injection (Supplementary Fig. 37-38). In contrast, CFA/IFA emulsions magnified the anti-OVA and

418 anti-PEG responses by $\sim 2$-fold. Notably, no change in the specificity and subtype of the immune response

419 developed towards the treatments was detected (Supplementary Fig. 37). In sum, this data confirmed the

420 immunogenicity benefits of POEGMA over PEG even delivered with a potent adjuvant.

421 Effect of ADAs on long-term efficacy. When immunogenicity and long-term efficacy data were evaluated 422 together, they suggested that the inferior blood glucose control with the increasing number of injections and poor $423 \mathrm{HbA1c} \%$ control by Ex-PEG $\mathrm{Rh}$ could result from anti-PEG antibody interference. To test this hypothesis, we next 424 measured the ADA levels in the plasma of long-term treated mice. To do so, plasma was collected on day 66 after 4258 weekly treatments and assayed for ADAs by the Luminex immunoassay. Both exendin and Bydureon induced a 426 mild anti-exendin IgM response. Notably, Ex-POEGMA ${ }_{o p t}$ did not induce anti-POEGMA antibodies and resulted in 427 a much weaker anti-exendin IgM response, possibly due to the non-immunogenic nature of POEGMA and the 428 shielding of exendin's immunogenic epitopes by POEGMA (Supplementary Fig. 39a). Notably, anti-exendin IgM 429 titers were higher in Ex-PEG ${ }_{R h}$ treated mice plasma, and an anti-exendin IgG response was detected 430 (Supplementary Fig. 39b). Ex-PEG ${ }_{R h}$ also induced both PEG-specific IgM and IgG responses. Given the absence of 431 exendin- and PEG-specific IgGs in the prior immunogenicity experiment, the observation of IgGs towards both 432 exendin and PEG in mice treated with Ex-PEG ${ }_{R h}$ was attributed to the greater number of injections ( 3 in the 
433 immunogenicity study vs. 8 here), more frequent dosing (17 vs. 7 days), and the longer time of the treatment (44 434 vs. 66 days) that are known to affect the immune response. ${ }^{42}$

We next investigated if the ADAs could neutralize the treatments. We analyzed long-term treated mice plasma for neutralizing antibodies (NAbs) using the cell-based assay developed to test the GLP1R-binding by exendin variants with minor modifications. ${ }^{44}$ Assay design and optimization are summarized in the Supplementary 438 Information and given in Supplementary Table 9 and Supplementary Fig. 40. The GLP1R-binding by the 439 treatments did not change when incubated with the mice plasma, indicating that the ADAs did not neutralize the treatments (Supplementary Fig. 41). This result was consistent with the results from the clinical trials of exendin, 441 where anti-exendin ADAs did not affect its PD. We also tested if PEG-specific ADAs neutralized Ex-PEG ${ }_{M w}$ by 442 incubating the conjugate with the plasma samples of the mice immunized with FA emulsion of OVA-PEG. Ex-PEG 443 showed no change in activity (Supplementary Fig. 41e), indicating that even high titers of anti-PEG antibodies did 444 not have neutralizing activity.

We finally tested the effect of ADAs on the PK of the treatments. We hypothesized that the loss of efficacy in the Ex-PEG $\mathrm{Mw}_{\mathrm{w}}$ long-term treatment was due to the binding of the anti-PEG antibodies to the circulating drug, 447 resulting in accelerated blood clearance and preventing it from showing efficacy. ${ }^{42,48}$ We also hypothesized that

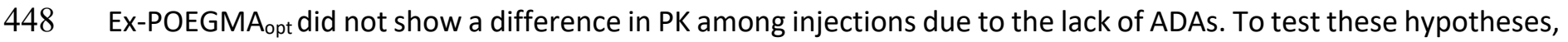
449 we s.c. administered sterile, endotoxin-free, and fluorescently-labeled exendin, Ex-PEG Rh, and Ex-POEGMA $_{\text {opt }}$ into 450 DIO C57BL/6J ( $n=4)$ and tracked their PK after the first (naïve mice) and fifth injection (immunized mice). Exendin 451 did not show any difference in PK parameters between naïve and immunized mice (Fig. 4c; Table 2), indicating 452 that anti-exendin antibodies were not PK-altering, consistent with the literature. ${ }^{44}$ Importantly, Ex-PEG Rh $_{\text {showed }}$ 453 significantly different PK profiles in naïve and immunized mice (Fig. 4d; Table 2). Its absorption was significantly 454 lower in the immunized mice, suggesting local lymph nodes' involvement in its elimination. ${ }^{49}$ Ex-PEG ${ }_{R h}$ 's 455 elimination half-life was $\sim 2$-fold shorter in the immunized mice ( $t_{1 / 2}$ elimination $=11.4 \pm 5.4 \mathrm{~h}$ vs. $\left.23.1 \pm 6.4 \mathrm{~h}\right)$, indicating 456 the PK-altering nature of anti-PEG antibodies. Together, the $\sim$-fold lower drug exposure in immunized mice 
( $A \cup C=424 \pm 35$ vs. $1585 \pm 112$ ) indicated that anti-PEG immune response was responsible for the loss of efficacy

458 in the long-term Ex-PEG ${ }_{R h}$ treatment. Notably, Ex-POEGMA $A_{o p t}$ preserved its PK benefits after repeated 459 administrations (Fig. 4e; Table 2), owing to its non-immunogenic structure.

460 Histopathological effects. We next investigated if treatment with Ex-POEGMA $A_{\text {opt }}$ had any histopathological effects

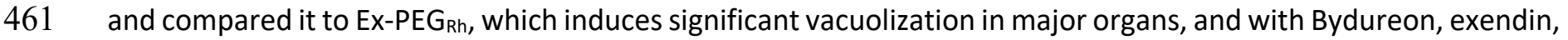
462 and PBS (- control). ${ }^{50}$ We collected kidney, pancreas, spleen, and thyroid tissues from the $d b / d b$ mice $(n=3)$ treated 463 in the long-term study. These tissues were fixed in 10\% neutral buffered formalin (NBF), processed, embedded 464 into paraffin, sectioned, and hematoxylin and eosin (H\&E) stained. The resulting tissue slides were evaluated by a 465 veterinary pathologist experienced in toxicologic pathology. Exendin and Bydureon treatment did not induce any 466 histopathological effects on the kidney (Supplementary Fig. 42a-c), whereas significant vacuolization was noted 467 in the Ex-PEG $\mathrm{Rh}$ group (Supplementary Fig. 42d). These vacuolar lesions were prominent primarily within epithelial 468 cells of the renal proximal tubules in the outer cortical region. Similarly, a low level of vacuolar change was also

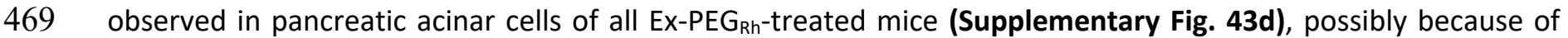
470 GLP1R targeting by exendin ${ }^{51}$ and vacuole formation by the conjugated PEG. No changes were noted within the 471 splenic or thyroid tissues treated with Ex-PEG (Supplementary Fig. 44). Importantly, Ex-POEGMA ${ }_{\text {opt }}$ treatment 472 induced no histopathological changes in the kidney (Supplementary Fig. 42e) or pancreas (Supplementary Fig. 473 43e).

474 Discussion

We have developed a PEG-like, minimally immunogenic, and injectable conjugate for sustained delivery 476 of biologics from a s.c. depot. This next-generation POEGMA technology platform has five key advantages over 477 traditional PEGylation. First, to date, PEG has been conjugated with biologics to create only soluble drug 478 formulation. The injectable, depot-forming POEGMA adds a new dimension to PEGylation by creating a drug depot 479 under the skin that achieves sustained drug release into the circulation. Synthesizing a POEGMA conjugate of 480 exendin that is injectable but undergoes an LCST phase transition into an insoluble coacervate phase that provides 
481 the maximal duration of release from the s.c. depot with a plasma concentration that remains above the 482 therapeutic threshold required a step-wise optimization of the polymer composition and $M_{w}$. In the first stage of 483 optimization, we fixed the $M_{w}$ of the POEGMA but varied the ratio of the EG2 and EG3 side chains in the monomer 484 to create a set of copolymers that exhibit a range of $T_{t}$. Upon s.c. injection of these Ex-POEGMA conjugates in 485 mice, we found that the $T_{t}$ of these copolymers determined the depot's stability, with depots with a lower $T_{t}$ 486 leading to more stable depots, but a slower rate of release, while depots with a higher $\mathrm{T}_{\mathrm{t}}$ had a higher rate of 487 release with a shorter duration of release. We identified $\sim 30^{\circ} \mathrm{C}$ as the optimal $\mathrm{T}_{\mathrm{t}}$ that balances the release rate of 488 the conjugate (and thereby steady-state plasma concentration) with the duration of release, so that a depot with 489 a $T_{t}$ of $\sim 30 \circ C$ provides the maximum efficacy, as by the blood glucose AUC. In the next optimization step, we fixed 490 the $T_{t}$ of the conjugate at $\sim 30 \circ \mathrm{C}$ while varying their $M_{w}$ from $\sim 20$ to $\sim 170 \mathrm{kDa}$. The rationale for this second stage 491 of polymer optimization was the recognition that once the conjugate is released from the s.c. depot, the plasma 492 half-life is controlled by renal clearance, which is a function of the $M_{w}$ of the conjugate. In vivo efficacy studies 493 revealed that a conjugate $M_{w}$ of $\sim 55$ Da yielded the maximum reduction in glucose AUC compared to the PBS 494 control. Together, these experiments identified an optimal Ex-POEGMA conjugate that has the highest efficacy, 495 with a $T_{t}$ of $\sim 30 \circ C$ and an $M_{w}$ of $\sim 55 \mathrm{kDa}$. preserving its favorable PK and PD benefits. The hyperbranched structure of POEGMA does not induce a POEGMA498 specific immune response, presumably because its short OEG side chains do not crosslink B-cell receptors as PEG 499 does, which is a prerequisite for T-cell independent B-cell immune responses. ${ }^{42}$ Unlike PEG, which is unstructured 500 in aqueous solvents, POEGMA self-organizes into nanoparticle-like chains due to the interfacial energy balance 501 driven by its amphiphilicity and the repulsion forces among hydrated OEG side-chains. ${ }^{45}$ We speculate that 502 POEGMA's compact molecular conformation in water may effectively reduce the number of epitopes exposed to 503 the immune system, allowing it to remain non-immunoreactive. Third, in addition to its lack of immunogenicity, 504 POEGMA does not react with PEG antibodies, suggesting that it can be safely used in the pre-existing PEG antibody 
positive population, which is now rampant because of chronic exposure to PEG through excipients in drugs and

506 consumer products. These findings are highly topical because up to $70 \%$ of the human population has pre-existing

507 antibodies to $\mathrm{PEG},{ }^{11}$ and billions of individuals are in the process of being immunized with a PEG-containing

508 liposome formulation of the Pfizer and Moderna vaccines for COVID-19. There is also a growing consensus that

509 PEG is responsible for the immediate-type hypersensitivity reactions ${ }^{52,53}$ seen with the Moderna and Pfizer COVID-

$510 \quad 19^{54}$ and other vaccines. ${ }^{55}$ Fourth, another favorable feature of POEGMA is that it does not exhibit the PEG-induced

511 vacuolization problem, presumably due to its partially biodegradable and non-immunogenic structure. We

512 speculate that cleavage of the OEG side chains from the polymethacrylate backbone through the ester bonds by

513 esterase enzymes in the blood ${ }^{56}$ reduces overall hydration, thereby conjugate size, allowing POEGMA to clear by

514 the kidneys. Finally, injectable and depot-forming POEGMA technology allows chromatography-free purification

515 of conjugated drugs, eliminating a significant time-, labor-, and cost-intensive step in manufacturing PEGylated

516 drugs.

Collectively, these advantages allowed an optimal Ex-POEGMA depot to provide T2D management superior to PEG and a clinical sustained-release exendin formulation-Bydureon. Importantly, Ex-PEG lost its 519 efficacy after repeated administrations due to the induction of PEG-specific PK-altering antibodies, preventing it 520 from being used as a long-term treatment strategy. In contrast, the optimal Ex-POEGMA conjugate preserved its 521 efficacy and PK benefits through repeated injections over 56 days, indicating that optimizing the molecular 522 architecture - as we have done with POEGMA - is a powerful approach to solve the mounting problems of 523 PEGylated therapeutics.

The POEGMA depot technology has several advantages over other drug delivery technologies. First, POEGMA combines sustained-release and extended circulation in one molecule. Most exendin formulations, such 526 as Bydureon, release the unmodified exendin peptide into the bloodstream that, after release, circulates only for 527 a few hours, while the POEGMA depot releases exendin as a POEGMA conjugate that has $~ 46$-fold longer half-life 528 in the circulation than the unmodified peptide. The molecular design of the POEGMA conjugate also eliminates 
529 the variable drug loading seen with PLGA ${ }^{57}$ - and nanoparticle-based formulations ${ }^{58}$ as exendin is covalently 530 conjugated with POEGMA at a known-equimolar-stoichiometry. In recent years, the Fc fusion technology531 wherein a peptide or protein drug is presented on an IgG Fc scaffold - has become increasingly popular because 532 it provides a long plasma half-life due to the Fc component of the fusion ${ }^{59}$ and because its manufacturing leverages 533 the established expertise of the bioprocessing industry in overexpression of antibody drugs in engineered 534 mammalian cell lines. However, the Fc fusion technology is not a panacea for all peptide and protein drugs that 535 have a short circulation time, as not all drugs can be expressed as Fc fusions because even subtle changes in the 536 Fc structure can dramatically alter the potency and function of the fused drug. ${ }^{59}$ In contrast to Fc fusions restricted 537 to genetically encodable peptides and proteins, the injectable POEGMA drug depot technology applies to all 538 biologics-peptide, protein, and oligonucleotide drugs that have suboptimal PK. The fact that the injectable 539 POEGMA depot technology is competitive with Fc fusion can be inferred by comparing the results presented 540 herein exendin with a preclinical study on Trulicity - a once-weekly FDA-approved Fc fusion of an exendin analog 541 GLP-1. Trulicity required twice-weekly injections in $d b / d b$ mice to adequately control blood glucose, whereas a

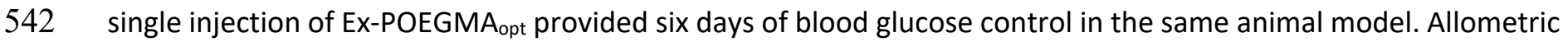
543 scaling suggests that Ex-POEGMA opt can be administered twice-monthly to T2D patients, ${ }^{60}$ necessitating less 544 frequent injections and thereby promoting better patient adherence, currently a major problem in diabetes 545 management. Third, a POEGMA depot releases a steady amount of drug into circulation to achieve a desired 546 therapeutic effect. In contrast, PLGA ${ }^{57}$ - and nanoparticle-based sustained-release exendin formulations ${ }^{58}$ have a 547 burst release profile, characterized by a peak-and-valley PK. ${ }^{61}$ Fourth, Ex-POEGMA conjugate eliminates the need 548 for surgical implantation, replacement, and removal seen with an exendin pump -TCA 650 (Intarcia), ${ }^{62}$ thereby 549 patient discomfort. Finally, POEGMA shields exendin's immunogenic epitopes, while free exendin-releasing 550 technologies do not have a mechanism for reduced immunogenicity, resulting in higher ADA titers and potentially 551 altered drug efficacy. Our results supported this assertion, where no anti-exendin ADAs were detected after long552 term treatment with Ex-POEGMA ${ }_{\text {opt}}$, while exendin and Bydureon treated mice had exendin-specific ADAs. 
In conclusion, the next-generation POEGMA technology offers a new and innovative approach to combat

554 the growing antigenicity and immunogenicity problems of PEG with a bonus of enhanced PK and PD benefits over

555 existing PEG conjugates. Future plans include investigating the biodistribution of POEGMA, identifying the 556 molecular variables that endow low immunogenicity to POEGMA conjugates, and testing the immunogenicity and 557 efficacy of POEGMA-drug conjugates in a large animal preclinical model as a prelude to clinical translation.

\section{Methods}

559 Synthesis and purification of POEGMA. All materials were purchased from Millipore Sigma. Triethylene glycol methyl ether methacrylate (EG3) and diethylene glycol methyl ether methacrylate (EG2) were passed through 561 basic alumina columns to remove inhibitors. Other materials were used as received. A catalytic complex was 562 prepared by mixing tris(2-pyridylmethyl) amine (TPMA) and copper (II) bromide ( $\left.\mathrm{CuBr}_{2}\right)$ in ultrapure water with 56318.2 MOhm resistivity at a final concentration of $0.8 \mathrm{M}$ and $0.1 \mathrm{M}$, respectively. In a typical copolymerization, a 564 Schlenk flask contained EG3 $(3.5 \mathrm{mmol} ; 701.57 \mu \mathrm{l})$, EG2 $(6.5 \mathrm{mmol} ; 1199.44 \mu \mathrm{l})$, azide functional polymerization 565 initiator (0.2 M in methanol; $125 \mu \mathrm{l}$; see Supplementary Information for synthesis), the catalytic complex (62.5 $566 \mu \mathrm{l})$, methanol $(5.875 \mathrm{ml})$ and $100 \mathrm{mM} \mathrm{NaCl}(11.946 \mathrm{ml})$. The polymerization flask was sealed and cooled to $0^{\circ} \mathrm{C}$ in 567 an ice bath. A separate Schlenk flask contained $64 \mathrm{mM}$ ascorbic acid in ultrapure water. Both flasks were purged 568 with argon for 45 min on ice to remove oxygen. After deoxygenation, the ascorbic acid solution was continuously 569 injected into the polymerization flask at a rate of $1 \mu \mathrm{min}^{-1}$ using a syringe pump under an inert atmosphere. The 570 resulting solution was kept under vacuum to remove methanol and freeze-dried overnight. The resulting POEGMA 571 was dissolved in acetonitrile and passed through a neutral alumina column to remove the catalytic complex. 572 POEGMA was purified from unreacted monomer by precipitation in cold diethyl ether, followed by overnight 573 evaporation of excess diethyl ether under vacuum.

574 Physical characterization of POEGMA. The $M_{n}, M_{w}$, and $\oslash$ of POEGMA were assessed by GPC-MALS. POEGMA was 575 solubilized in tetrahydrofuran (THF) at $2 \mathrm{mg} \mathrm{ml}^{-1}$, followed by filtration through a $0.22 \mu \mathrm{m}$ Teflon syringe filter. 50 $576 \mu$ l of the solution was separated on an Agilent PLgel mixed-C column (105 $\AA$, $7.5 \mathrm{~mm}$ internal diameter x $300 \mathrm{~mm}$ 
length, and $5 \mu \mathrm{m}$ particle size) using an Agilent 1100 analytical high-pressure liquid chromatography (HPLC). The

578 HPLC was equipped with a UV detector operating at $254 \mathrm{~nm}$ (Agilent), a Dawn EOS MALS detector (Wyatt

579 Technology), and an Optilab DSP refractometer (Wyatt Technology). The mobile phase consisted of $100 \mathrm{ppm}$

580 butylated hydroxytoluene (BHT) in THF as a stabilizer at a $1 \mathrm{ml} \mathrm{min}^{-1}$ flow rate. Before each analysis, the MALS

581 detector was normalized with $30 \mathrm{kDa}$ polystyrene (Wyatt Technology). Refractive index increment (dn/dc) of

582 POEGMA was calculated using ASTRA software provided with the HPLC (v. 6.0, Wyatt Technology) based on

583 injections of known concentrations and mass, followed by data analysis for $M_{n}, M_{w}$, and $\oslash$.

584 Structural characterization of POEGMA. The POEGMA structure was characterized by H-NMR spectroscopy using 585 a $400 \mathrm{MHz}$ Varian INOVA spectrometer and ACD/NMR software (ACD Labs). The monomer composition was 586 defined as the molar percentage of EG2 (or EG3) content in the copolymer. The monomer composition was 587 calculated from the integral value that corresponds to the average number of hydrogens $(\mathrm{H})$ present in the OEG 588 side-chain (b; 3.4-4.4 ppm; $6 \mathrm{H}$ for $E G 2_{100 \%}$ homopolymer; $10 \mathrm{H}$ for $E \mathrm{EG}_{100 \%}$ homopolymer) except the chain end589 group (c; 3.5-3.3 ppm; $3 \mathrm{H}$ ) and methylene protons (a; $4.0-4.4 \mathrm{ppm} ; 2 \mathrm{H})$. The DP were calculated by subtracting 590 the polymerization initiator's $M_{\mathrm{w}}$ from POEGMA's $M_{\mathrm{w}}$ and dividing the resulting mass by the average $M_{\mathrm{w}}$ of a 591 monomeric unit.

592 Hydrodynamic size characterization. The $R_{h}$ of POEGMA and Ex-POEGMA conjugates were characterized by 593 dynamic light scattering (DLS) in PBS at $1 \mathrm{mg} \mathrm{ml}^{-1}$ using a temperature-controlled DynaPro Plate Reader (Wyatt 594 Technology). Samples were filtered through a $100 \mathrm{~nm}$ syringe filter (Whatman) prior to measurement. Ten repeat 595 measurements of 10 -second acquisitions were made at $15^{\circ} \mathrm{C}$. Data were analyzed for Raleigh spheres by applying 596 a regularization fit using Dynamics 6.12.0.3 software (Wyatt Technology). The laser wavelength and scattering 597 angle of the instrument were $831.95 \mathrm{~nm}$ and $90^{\circ}$, respectively.

598 Phase behavior characterization. The optical density of POEGMA and Ex-POEGMA conjugates were monitored at $599600 \mathrm{~nm}$ in PBS at $\mathrm{pH} 7.4$, as the temperature of the cuvet containing the samples was increased at a rate of $1^{\circ} \mathrm{C}$ $600 \mathrm{~min}^{-1}$ on a temperature-controlled UV-vis spectrophotometer (Cary 300 Bio, Varian Instruments). A sharp increase 
601 in the optical density with temperature indicates the occurrence of the phase transition. The $T_{t}$ was defined as the 602 temperature at the inflection point of the optical density versus the temperature curve and is calculated as the 603 maximum of the first derivative using GraphPad Prism 8.0 software. The reversibility of the phase behavior was 604 investigated by monitoring the optical density as the temperature was gradually decreased from a temperature 605 above the $T_{t}$ back to $15 \circ \mathrm{C}$.

606 Protein expression and purification. Exendin was recombinantly expressed in E. coli from a synthetic gene in a 607 pMA-T expression vector as an ELP fusion protein with a sortase-A recognition site (LPETG) and a polyhistidine 608 tag, yielding exendin-LPETG-His ${ }_{6}$-ELP (ESE). The ELP tag enables rapid non-chromatographic purification of ESE, 609 while the LPETG peptide acts as the sortase ligation site for the ATRP initiator. Both ESE and His 6 -Sortase A were 610 available from a previous study and expressed and purified to $>95 \%$ purity, as previously described..$^{18}$ The purity 611 of all proteins was visualized by sodium dodecyl sulfate-polyacrylamide gel electrophoresis (SDS-PAGE) using 4612 20\% precast Tris-HCl gels (Bio-Rad), followed by staining with Simply Blue Safe Stain (Thermo Scientific) and gel 613 densitometry analysis using Image Lab software (Bio-Rad). The ESE concentration was measured using a 614 Bicinchoninic Acid (BCA) assay (Pierce) according to the manufacturer's instructions. The His 6 -Sortase A 615 concentration was measured by UV-visible spectroscopy on an ND-1000 Nanodrop spectrophotometer (Thermo 616 Scientific) and the known extinction coefficient of the protein $\left(14440 \mathrm{M}^{-1} \mathrm{~cm}^{-1}\right)$.

617 Synthesis, purification, and characterization of exendin-DBCO. A bio-orthogonal DBCO group was installed on 618 the C-terminus of exendin by sortase A-mediated native peptide ligation, yielding exendin-DBCO. Briefly, ESE (100 $619 \mu \mathrm{M})$ and $\mathrm{His}_{6}$-Sortase $\mathrm{A}(50 \mu \mathrm{M})$ were reacted in the presence of triglycine-DBCO (Gly $\left.{ }_{3}-\mathrm{DBCO}\right)(5 \mathrm{mM}$; Click 620 Chemistry Tools) in ligation buffer (50 mM Tris, $150 \mathrm{mM} \mathrm{NaCl}$, and $10 \mathrm{mM} \mathrm{CaCl}_{2} ; \mathrm{pH} 7.5$ ) at room temperature for 62116 hours. Exendin-DBCO was purified from the reaction mixture by reverse immobilized metal affinity 622 chromatography using an AKTA Purifier (GE Healthcare) equipped with a photodiode array operating at 220 and $623280 \mathrm{~nm}$ and a HisTrap HP (GE Healthcare) column. Exendin-DBCO was collected in the flow-through as it was the 624 only species that did not carry an oligohistidine tag, and hence did not bind to the resin. Exendin-DBCO was 
625 concentrated by ultrafiltration using Centricon 70 (Millipore Sigma) filters with a 3,000 Da molecular weight cut626 off (MWCO), followed by dialysis into cold water and lyophilization. Stoichiometric (1:1) attachment of DBCO to 627 exendin was confirmed by Matrix-Assisted Laser Desorption/lonization-Time-of-Flight mass spectroscopy (MALDI628 TOF-MS).

629 Synthesis and purification of exendin conjugates. Exendin-DBCO was conjugated to azide-functional POEGMA or 630 PEG via the strain-promoted alkyne-azide click reaction. Exendin-DBCO and azide-functional POEGMA or PEG were 631 dissolved in PBS at a 1.05:1 ratio and reacted overnight at $4^{\circ} \mathrm{C}$. Depot-forming Ex-POEGMA conjugates were 632 purified by triggering their LCST phase transition by the addition of ammonium sulfate to a final concentration of $633 \quad 0.1 \mathrm{M}$. The dense phase that contained the conjugate was recovered by centrifugation at $21,000 \mathrm{~g}$ for 15 minutes 634 at room temperature, the supernatant was removed, and the conjugate was dissolved in PBS at 4으. The last two 635 steps were repeated twice to obtain conjugates with > 99\% purity, as verified by SDS-PAGE and HPLC. In the final 636 step, the conjugate was dissolved in ultra-pure water and lyophilized. Soluble Ex-POEGMA and Ex-PEG conjugates 637 were purified by a single round of size exclusion chromatography (SEC) using an AKTA purifier equipped with a 638 photodiode array detector operating at 220 and $280 \mathrm{~nm}$ and a HiLoad 16/600 Superdex 75 pg column (GE 639 Healthcare) at 4 으 $\mathrm{C}$ using PBS as the mobile phase. The purified conjugates were concentrated by ultrafiltration 640 using Amicon filters (Millipore Sigma) with a 3,000 Da MWCO, followed by dialysis into the water at 4ㅇ overnight 641 and lyophilization.

642 Physical characterization of exendin conjugates. The conjugates were characterized in terms of their $M_{n}, M_{w}$, 643 and $\oplus$ by SEC-MALS using an Agilent 1260 analytical HPLC equipped with a UV detector operating at $280 \mathrm{~nm}$ 644 (Agilent), a DAWN HELEOS II MALS detector (Wyatt Technology), and an Optilab T-rEX refractive index detector 645 (Wyatt Technology). The DAWN HELEOS II MALS detector was annually calibrated in toluene and normalized with $6462 \mathrm{mg} \mathrm{ml}^{-1}$ bovine serum albumin (Pierce) before each analysis. The exendin conjugates were dissolved in $10 \mathrm{mM}$

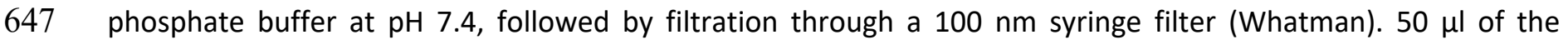
648 conjugate was separated on a Shodex KW-803 column ( $8 \mathrm{~mm}$ internal diameter $\times 300 \mathrm{~mm}$ length, and $5 \mu \mathrm{m}$ particle 
size) at a flow rate of $0.5 \mathrm{ml} \mathrm{min} \mathrm{m}^{-1}$. The mobile phase was $30 \%(\mathrm{v} / \mathrm{v})$ methanol in $10 \mathrm{mM}$ phosphate buffer at $\mathrm{pH}$

7.4. Data were analyzed for $M_{n}, M_{w}$, and $\emptyset$ using ASTRA software (v. 7.0, Wyatt Technology).

651 In vitro activity of exendin variants. The activity of exendin and its conjugates with POEGMA and PEG were 652 measured by GLP1R activation in a cell-based assay, which increases intracellular cyclic adenosine 653 monophosphate (CAMP) levels upon activation of the GLP1R by exendin or its conjugates. Intracellular cAMP 654 concentrations were quantified by treating Human Embryonic Kidney 293 cells recombinantly expressing GLP1R 655 and luciferase fused CAMP (HEK293/CRE-Luc/GLP1R) with exendin variants. This recombinant cell line was a 656 generous gift from Timothy Kieffer (University of British Columbia) and authenticated via short tandem repeat 657 (STR) analysis by the American Type Culture Collection (ATCC). HEK293/CRE-Luc/GLP1R cells were cultured in high glucose Dulbecco's Minimal Essential Medium (DMEM) (Gibco), supplemented with 10\% fetal bovine serum (Hyclone), $400 \mu \mathrm{g} \mathrm{m}^{-1} \mathrm{G} 418$ (Thermo Fisher), and $660200 \mu \mathrm{g} \mathrm{ml}^{-1}$ Hygromycin B (Invitrogen). Cells were subcultured at least once before the assay at approximately $66180 \%$ confluency. One day before performing the assay, cells were seeded without antibiotics in phenol red-free DMEM (Gibco) on 96 -well plates at 25,000 cells per well in $90 \mu \mathrm{l}$ media followed by incubation at $37^{\circ} \mathrm{C}$ under $5 \%$ 663 CO2 atmosphere overnight. Exendin conjugates (20 $\mu \mathrm{M}$ in PBS) were incubated with dipeptidyl peptidase IV (DPP664 IV, Prospec Bio) to expose an active $\mathrm{N}$-terminus for $16 \mathrm{~h}$ at room temperature. DPP-IV amount was 2.5 mass\% of 665 exendin present in the conjugates. On the day of the assay, exendin (Santa Cruz Biotechnology) was dissolved to 666 a final concentration of $20 \mu \mathrm{M}$ in PBS. Logarithmic serial dilutions were made for exendin variants in PBS using 667 unmodified exendin as a positive control. $10 \mu \mathrm{l}$ of each dilution was transferred to wells $(n=6)$, yielding a 668 concentration range of $10^{-13}-10^{-6} \mathrm{M}$. The plates were then incubated at $37^{\circ} \mathrm{C}$ for 5 hours, followed by equilibration 669 with room temperature for 1 hour. Next, $100 \mu \mathrm{l}$ Bright-Glo ${ }^{\mathrm{TM}}$ reagent (Promega) was added to the wells and 670 incubated for 2 minutes, followed by measuring luminescence using a Victor plate reader (Perkin Elmer). Data 671 were analyzed for net luminescence by subtracting PBS-treated wells' mean luminescence (negative control) from 
672 exendin variants. The effective half-maximal dose $\left(E C_{50}\right)$ of each exendin variant was determined by fitting the 673 dose-response curve to a four-parameter logistic, nonlinear regression model using GraphPad Prism 8 software.

674 In vivo studies. In vivo studies were conducted under protocols approved by Duke Institutional Animal Care and 675 Use Committee (IACUC) by employing six-week-old male C57BL/6J (Jackson Laboratories; stock no. 000664) or 676 B6.BKS(D)-LepR ${ }^{d b} / J$ mice ( $d b / d b$; Jackson Laboratories; stock no: 000697). C57BL/6J mice were kept on a 60 677 kilocalorie (kcal) \% fat diet (Research Diets Inc.; \#D12492i) for at least five weeks before and during the 678 experiments unless otherwise noted, yielding DIO mice. Six-week-old male $d b / d b$ mice were fed a standard rodent 679 diet (LabDiet 5001) and acclimatized to facilities for one week before the experiments. In immunogenicity 680 experiments, mice treated with OVA variants were kept on a standard rodent diet. Mice were group-housed under 681 controlled photoperiod with $12 \mathrm{~h}$ light and $12 \mathrm{~h}$ dark cycles and acclimated to the facility for a week before the 682 start of experiments. Mice had ad libitum access to water and food unless otherwise noted. $\mu \mathrm{m}$ Acrodisc filter with a Mustang E membrane (Pall Corporation). The final endotoxin amount was tested below 6855 EU per kg mouse body weight using the Endosafe nexgen-PTS instrument and cartridges (Charles River). For the 686 samples used in the immunogenicity and the long-term efficacy experiments, a more stringent endotoxin limit of 687 a maximum of $0.2 \mathrm{EU}$ per kg mouse body weight was used.

688 Fed blood glucose measurements. In the short-term efficacy experiments, the fed blood glucose was measured 689 after a single s.c. injection of the treatments. On the day of injection, the tail was sterilized with alcohol pads (BD). 690 The first drop of blood collected from a tiny incision on the tail vein was wiped off. The second drop of blood was 691 used to measure fed blood glucose using a hand-held glucometer (AlphaTrack, Abbott). The treatments were 692 solubilized in PBS and kept on ice before injection to prevent phase transition, followed by s.c. administration into 693 mice. Bydureon was prepared for injection according to the manufacturer's instructions. Fed blood glucose levels 694 were measured $24 \mathrm{~h}$ and immediately before injection, at 1-, 4-, and 8-h post-injection, and every $24 \mathrm{~h}$ after that 
695 until no significant effect of treatments on fed blood glucose was observed. Body weight was tracked daily. In the 696 long-term efficacy experiment, fed blood glucose and body weight were tracked every three days.

697 IPGTT. The glycemic regulation ability of treatments was assessed by performing three IPGTTs after a single s.c. 698 injection. $\mathrm{Db} / \mathrm{db}$ mice were used during the first week of the long-term pharmacodynamics study. On day 0 , the 699 treatments were administrated into the mice at an equivalent dose (1000 nmol per $\mathrm{kg}$ bodyweight) and 700 concentration $(500 \mu \mathrm{M})$ using the equivalent injection volume of PBS as a negative control. Bydureon was 701 prepared for injection according to the manufacturer's instructions. On the day of the IPGTT (Day 1, 3, and 5), 702 mice were fasted six hours before the glucose challenge by an i.p injection of $1.5 \mathrm{~g} \mathrm{~kg}^{-1}$ of glucose (Sigma) followed 703 by blood glucose monitoring at 5-, 15-, 30-, 60-, 90-, 120-, 180- and $240 \mathrm{~min}$.

$704 \quad H b A 1 c \%$ measurement. In long-term pharmacodynamics experiments, $d b / d b$ mice $(n=5)$ were repeatedly injected 705 with the treatments at an equivalent dose (1000 nmol per kg body weight in PBS) and concentration (500 $\mu \mathrm{M})$ 706 every seven days over 56 days using an equivalent injection volume of PBS as a negative control. Bydureon was 707 prepared for injection according to the manufacturer's instructions. HbA1c\% was measured on Day 0 before 708 injection, Day 28, and Day 56 using DCA Vantage Analyzer and DCA HbA1c reagent kit (Siemens).

709 Pharmacokinetics. Exendin variants were labeled with a fluorophore to track their pharmacokinetics. Briefly, 710 Alexa Fluor 488 NHS ester (Pierce) was reacted with exendin variants $\left(5 \mathrm{mg} \mathrm{ml}^{-1}\right)$ at a $5: 1$ molar ratio in PBS for 711 one hour at room temperature. Unreacted excess fluorophore was removed by dialysis into the water at $4^{\circ} \mathrm{C}$ using 712 membranes with a 3,000 Da MWCO (Pierce), verified by HPLC. The labeling efficiency was calculated from UV-vis 713 spectroscopy using an ND-1000 Nanodrop spectrophotometer (Thermo Scientific). The fluorophore-labeled treatments were administered into DIO C57BL/6J mice via a single s.c. injection 715 at $1000 \mathrm{nmol} \mathrm{kg}^{-1}\left(45 \mathrm{nmol} \mathrm{kg}^{-1}\right.$ fluorophore). Ten $\mu \mathrm{l}$ of blood was collected from a tiny incision on the tail vein into 716 tubes containing $90 \mu \mathrm{l}$ of 1,000 U ml-1 heparin (Sigma) at 5-min, 1-, 2-, 4-, 8-, 24-, 48-, 72-, 96-, 120-, 144- and 168717 h. Blood samples were centrifugated at $1600 \mathrm{~g}$ at $4^{\circ} \mathrm{C}$ for 15 minutes for plasma. Fluorophore concentration in 
718 plasma samples was detected by a Victor plate reader (Perkin Elmer) at $485 \mathrm{~nm}$ (excitation) and $535 \mathrm{~nm}$

719 (emission). PK parameters were derived by plotting the drug's plasma concentration as a function of time and 720 fitting it to a non-compartmental PK model for the absorption and elimination phases using GraphPad Prism 721 software. The absorption phase described the time between injection at $0-\mathrm{h}$ and $t_{\max }$, where the drug 722 concentration was maximum. The elimination phase described the time after $t_{\max } \cdot t_{\max }$ was calculated from 723 equation $t_{\max }=\left(\frac{2.303}{k_{a}-k_{e}}\right) * \log \frac{k_{a}}{k_{e}}$, where $k_{a}$ and $k_{e}$ were the apparent absorption and elimination rate constants. 724 The rate constants were determined from the linear regression slope of the log (drug concentration) versus time 725 graph using equation $k=-2.303 *$ slope. Half-lives of treatments in each phase were determined from the 726 equation $t_{1 / 2}=\frac{0.693}{k}$. The maximum drug concentration $\left(C_{\max }\right)$ was calculated at $t_{\max }$. Minimal effective 727 conjugate concentration was calculated by triangulating the concentration values based on the duration of blood 728 glucose control determined in short-term efficacy experiments in DIO mice.

ADAs' effect on the PK was investigated by weekly administering the fluorophore-labeled treatments into 730 the DIO C57BL/6J mice five times. Blood samples were collected after the first and last injection, and PK 731 parameters were determined as described above.

732 Immunogenicity. We tested the immunogenicity of POEGMA and compared it to that of PEG in three sets of 733 immunogenicity experiments. In the first set, sterile and endotoxin-free exendin, Ex-PEG $\mathrm{Mw}_{\mathrm{w}}$, and Ex-POEGMA 734 were s.c. administrated into 7-week-old C57BL/6J mice $(n=10)$ at an equivalent dose $\left(1000 \mathrm{nmol}^{\mathrm{kg}}{ }^{-1}\right)$ and 735 concentration $(500 \mu \mathrm{M})$. In the second set of experiments, mice were injected with OVA (Invivogen), OVA-PEG $10 \mathrm{~K}$, 736 and OVA-POEGMA $10 \mathrm{~K}$ at $9.6 \mathrm{nmol} \mathrm{kg}^{-1}(100 \mu \mathrm{l})$. In the final set, OVA, OVA-PEG $10 \mathrm{k}$, and OVA-POEGMA $10 \mathrm{~K}$ were 737 emulsified in an equal volume of sterile CFA/IFA (Invivogen) and then administrated into 7-week-old C57BL/6J 738 mice $(n=10)$ at $9.6 \mathrm{nmol} \mathrm{kg}^{-1}(100 \mu \mathrm{l})$. CFA was used for the first injections, while IFA was used for the subsequent 739 injections. An equal injection volume of PBS (or PBS emulsified in CFA or IFA) was used as a negative control in all 740 experimental sets. All drugs were administrated into mice every 17 days three times (Day 0,17 , and 34 ). The 
741 injection site was the loose skin over the shoulders and was kept the same across the injections. Blood samples 742 were collected seven days before the first injection (Day -7) and ten days after each injection (Day 10, 27, and 44), 743 followed by plasma isolation via centrifugation at $4^{\circ} \mathrm{C}$ at $1600 \mathrm{~g}$ for 15 minutes, aliquoting on ice, and storage at $74480^{\circ} \mathrm{C}$ until analysis.

745 Analysis of ADA response. ADAs were analyzed using a Luminex multiplex immunoassay according to the 746 manufacturer's instructions with minor modifications. Assay design, optimization, and validation were given in 747 Supplementary Information. The plasma samples of mice treated with exendin variants were diluted 200-fold in 0.2\% (w/v) I-Block protein-based blocking reagent (Thermo Scientific) in PBS (Hyclone), defined as the assay buffer. 749 The plasma samples of mice treated with OVA variants $(n=10)$ were diluted 500 - and 10000 -fold in the assay buffer 750 for IgM and IgG analysis, respectively.

In a typical LMI assay, $50 \mu$ l of the diluted plasma samples $(n=3)$ were transferred to a black, round-bottom 752 96-well-plate (Corning). Next, spectrally distinct exendin-, Ex-PEG-, Ex-POEGMA-, OVA-, OVA-PEG-, and OVA753 POEGMA-coupled magnetic bead sets were mixed at a concentration of 2500 beads per $50 \mu l$ per set. $50 \mu l$ of the 754 resulting solution was distributed to the wells of a 96-well plate and incubated for 1 hour on an orbital shaker to 755 capture ADAs present in the plasma samples. After incubation, 96-well-plate was placed on a magnetic ring stand 756 (Invitrogen) and incubated for 60 seconds for separation to occur. The supernatant was discarded, and wells were 757 washed with $100 \mu$ l of the assay buffer twice. The resulting magnetic bead-ADA complexes were incubated with $758100 \mu \mathrm{l}$ of $5 \mathrm{\mu g} \mathrm{ml}^{-1} \mathrm{R}$-Phycoerythrin-conjugated goat anti-mouse IgG (Jackson Immunoresearch; \#115-115-164) for 75930 minutes to detect IgG subtypes of ADAs. $100 \mu \mathrm{l}$ of $5 \mu \mathrm{g} \mathrm{ml} \mathrm{l}^{-1}$ biotin-conjugated goat anti-mouse IgM (Jackson 760 Immunoresearch; \#115-065-075) was used to detect IgM subtypes of ADAs, followed by washes, incubation with $761100 \mu \mathrm{l}$ of $7.5 \mathrm{\mu g} \mathrm{ml}^{-1}$ streptavidin-R-phycoerythrin (SAPE; Invitrogen) for 30 minutes, and a final round of washes. 762 Mouse IgG- or IgM-coupled magnetic beads were used as positive controls. They were incubated with $100 \mu$ l of $7630.25 \mathrm{\mu g} \mathrm{ml}^{-1} \mathrm{R}$-Phycoerythrin-conjugated goat anti-mouse IgG or $0.114 \mu \mathrm{g} \mathrm{ml}{ }^{-1}$ biotin-conjugated goat anti-mouse $764 \mathrm{IgM}$, followed by $0.171 \mathrm{\mu g} \mathrm{m}^{-1} \mathrm{SAPE}$. The negative control was the drug-coupled bead mixture in the assay buffer. 
765 Finally, magnetic beads were suspended in $100 \mu$ l of assay buffer, followed by measuring mean fluorescence signal 766 intensity (MFI) using MAGPIX (Luminex) instrument. The average MFI value of each plasma sample and treatment 767 was computed with no exception for outliers. Mouse plasma samples were considered ADA-positive if the average 768 MFI for any given bead type was above the background (see Supplementary Information). MFI of each plasma 769 sample for any given bead type was normalized to the positive control.

770 Histology. The $d b / d b$ mice used in the long-term efficacy experiment were sacrificed, followed by collecting the 771 organs into $10 \%$ neutral-buffered formalin. After fixation in $10 \% \mathrm{NBF}$, the tissues were trimmed, paraffin 772 embedded, and sectioned at $5 \mu \mathrm{m}$ for light microscopy, followed by H\&E staining.

773 Statistical Analyses. In all the animal studies, animals were randomized, and group sizes were estimated using 774 open-source G*Power software based on the literature, and adequate power was ensured to detect differences. 775 Data were presented as mean \pm standard error of the mean (SEM) unless otherwise noted differently. Blood 776 glucose data were plotted as a function of time using raw values and normalized values. Normalization reflects 777 the percent change in blood glucose with treatment. It was calculated by dividing raw the fed blood glucose 778 measurement at any given time point by an average value of fed blood glucose levels measured $24 \mathrm{hours}(\mathrm{t}=-24 \mathrm{~h})$ 779 and immediately before injection ( $\mathrm{t}=\mathrm{0h}$ ), as previously described in the literature. ${ }^{18}$ Blood glucose and body weight 780 data were analyzed using two-way repeated-measures analysis of variance (ANOVA) followed by post hoc 781 Dunnett's multiple comparison test. The AUC for glucose exposure was quantified for each subject using the 782 trapezoid rule and analyzed using two-way ANOVA, followed by post-hoc Tukey's multiple comparison test. $783 \mathrm{HbA} 1 \mathrm{c} \%$ and immunogenicity data were analyzed using two-way ANOVA, followed by post-hoc Tukey's multiple 784 comparison test. A test was considered significant if the $p$-value is $<0.05\left({ }^{*} p<0.05 ;{ }^{* *} p<0.01 ;{ }^{* *} p<0.001\right.$; $785 * * * * p<0.0001 ; \mathrm{ns}: p>0.05)$. GraphPad Prism 8.0 was used for all statistical analyses.

786 Acknowledgments. This work was funded by the National Institutes of Health (R01DK124276-01 to A.C). We thank 787 Dr. Stephen Craig at the Department of Chemistry for allowing us to use his GPC-MALS facilities to analyze 788 POEGMAs and Dr. Peter Silinski at the Duke Chemistry Mass Spectrometry Facility for mass spectrometry analyses 
on the monomers and the polymerization initiator. We also thank the Duke University School of Medicine for the use of the Proteomics and Metabolomics Shared Resource, which provided analysis on the stoichiometry of DBCO installment to exendin, and Duke Immune Profiling Core (DIPC) at Duke University Center for AIDS Research (CFAR)

792 for the use of Luminex MAGPIX.

793 Author Contributions. I.O. and A.C. conceived and designed the research. I.O., A.S., N.Z., and X.L. performed the 794 experiments. J.I.E. imaged the histology slides and interpreted the findings. J.H.C., J.E.C., and D.A.D. and 795 contributed to experimental design. I.O. and A.C. interpreted the results and wrote the manuscript. All authors 796 participated in the discussion of the data and commented on the manuscript.

797 Competing Interests. I.O. and A.C. have a pending patent on a stimuli-responsive PEG-like polymer-based drug 798 delivery platform (U.S. 17/051,202) and a U.S. provisional patent application on non-immunogenic PEG-like 799 polymer conjugate technology (U.S. 63/169,541).

800 Data Availability. All data is available in the manuscript or the supplementary materials.

$801 \quad$ References

8021 Leader, B., Baca, Q. J. \& Golan, D. E. Protein therapeutics: a summary and pharmacological classification. $803 \quad$ Nature Reviews Drug Discovery 7, 21-39, (2008).

8042 Fosgerau, K. \& Hoffmann, T. Peptide therapeutics: current status and future directions. Drug Discovery 805 Today 20, 122-128, (2015).

8063 Turner, M. R. \& Balu-lyer, S. V. Challenges and Opportunities for the Subcutaneous Delivery of Therapeutic 807 Proteins. Journal of Pharmaceutical Sciences 107, 1247-1260, (2018).

8084 Vugmeyster, Y., Xu, X., Theil, F. P., Khawli, L. A. \& Leach, M. W. Pharmacokinetics and toxicology of 809 therapeutic proteins: Advances and challenges. World J Biol Chem 3, 73-92, (2012).

8105 Ekladious, I., Colson, Y. L. \& Grinstaff, M. W. Polymer-drug conjugate therapeutics: advances, insights and 811 prospects. Nature Reviews Drug Discovery 18, 273-294, (2019). 
8147 Ganson, N. J., Kelly, S. J., Scarlett, E., Sundy, J. S. \& Hershfield, M. S. Control of hyperuricemia in subjects 815 with refractory gout, and induction of antibody against poly(ethylene glycol) (PEG), in a phase I trial of 816 subcutaneous PEGylated urate oxidase. Arthritis Res Ther 8, R12, (2006).

8178 Hershfield, M. S. et al. Induced and pre-existing anti-polyethylene glycol antibody in a trial of every 3818 week dosing of pegloticase for refractory gout, including in organ transplant recipients. Arthritis Res Ther 819 16, R63, (2014).

8209 Garay, R. P., El-Gewely, R., Armstrong, J. K., Garratty, G. \& Richette, P. Antibodies against polyethylene 821 glycol in healthy subjects and in patients treated with PEG-conjugated agents. Expert Opin Drug Deliv 9, 822 1319-1323, (2012).

82310 Richter, A. W. \& Akerblom, E. Polyethylene glycol reactive antibodies in man: titer distribution in allergic 824 patients treated with monomethoxy polyethylene glycol modified allergens or placebo, and in healthy $825 \quad$ blood donors. Int Arch Allergy Appl Immunol 74, 36-39, (1984).

82611 Yang, Q. et al. Analysis of Pre-existing IgG and IgM Antibodies against Polyethylene Glycol (PEG) in the 827 General Population. Analytical Chemistry 88, 11804-11812, (2016).

82812 Ganson, N. J. et al. Pre-existing anti-polyethylene glycol antibody linked to first-exposure allergic reactions 829 to pegnivacogin, a PEGylated RNA aptamer. J Allergy Clin Immunol 137, 1610-1613 e1617, (2016).

83013 Armstrong, J. K. et al. Antibody against poly(ethylene glycol) adversely affects PEG-asparaginase therapy 831 in acute lymphoblastic leukemia patients. Cancer 110, 103-111, (2007).

83214 Verhoef, J. J., Carpenter, J. F., Anchordoquy, T. J. \& Schellekens, H. Potential induction of anti-PEG 833 antibodies and complement activation toward PEGylated therapeutics. Drug Discov Today 19, 1945-1952, 834 (2014). 
83515 Zhang, P., Sun, F., Liu, S. \& Jiang, S. Anti-PEG antibodies in the clinic: Current issues and beyond PEGylation. 836 Journal of controlled release : official journal of the Controlled Release Society 244, 184-193, (2016).

83716 Fee, C. J. Size comparison between proteins PEGylated with branched and linear poly(ethylene glycol) 838 molecules. Biotechnology and Bioengineering 98, 725-731, (2007).

83917 Webster, R. et al. in PEGylated Protein Drugs: Basic Science and Clinical Applications (ed Francesco M. 840 Veronese) 127-146 (Birkhäuser Basel, 2009).

84118 Qi, Y. et al. A brush-polymer/exendin-4 conjugate reduces blood glucose levels for up to five days and 842 eliminates poly(ethylene glycol) antigenicity. Nature Biomedical Engineering 1, 0002, (2016).

84319 Joh, D. Y. et al. Architectural Modification of Conformal PEG-Bottlebrush Coatings Minimizes Anti-PEG 844 Antigenicity While Preserving Stealth Properties. Advanced Healthcare Materials 8, 1801177, (2019).

84520 Lu, L. et al. In vitro and in vivo characterization of a novel long-acting GLP-1 receptor agonist, exendin-4$846 \quad$ Fc fusion protein. RSC Advances 7, 54178-54187, (2017).

84721 Glaesner, W. et al. Engineering and characterization of the long-acting glucagon-like peptide-1 analogue 848 LY2189265, an Fc fusion protein. Diabetes/Metabolism Research and Reviews 26, 287-296, (2010).

84922 Zhang, B., Tang, H. \& Wu, P. In Depth Analysis on the Unusual Multistep Aggregation Process of 850 Oligo(ethylene glycol) Methacrylate-Based Polymers in Water. Macromolecules 47, 4728-4737, (2014).

85123 Sun, S. \& Wu, P. On the Thermally Reversible Dynamic Hydration Behavior of Oligo(ethylene glycol) 852 Methacrylate-Based Polymers in Water. Macromolecules 46, 236-246, (2013).

85324 Badi, N. Non-linear PEG-based thermoresponsive polymer systems. Progress in Polymer Science 66, 54$85479,(2017)$.

85525 Luginbuhl, K. M. et al. One-week glucose control via zero-order release kinetics from an injectable depot 856 of glucagon-like peptide-1 fused to a thermosensitive biopolymer. Nat Biomed Eng 1, (2017).

85726 Simakova, A., Averick, S. E., Konkolewicz, D. \& Matyjaszewski, K. Aqueous ARGET ATRP. Macromolecules $858 \quad 45,6371-6379,(2012)$. 
85927 Ramírez-Jiménez, A., Montoya-Villegas, K. A., Licea-Claverie, A. \& Gónzalez-Ayón, M. A. Tunable Thermo860 Responsive Copolymers from DEGMA and OEGMA Synthesized by RAFT Polymerization and the Effect of 861 the Concentration and Saline Phosphate Buffer on its Phase Transition. Polymers (Basel) 11, 1657, (2019). 86228 Lutz, J.-F. Polymerization of oligo(ethylene glycol) (meth)acrylates: Toward new generations of smart 863 biocompatible materials. Journal of Polymer Science Part A: Polymer Chemistry 46, 3459-3470, (2008).

86429 Ozer, I. \& Chilkoti, A. Site-Specific and Stoichiometric Stealth Polymer Conjugates of Therapeutic Peptides 865 and Proteins. Bioconjugate Chemistry, (2016).

86630 Pang, Y., Liu, J., Qi, Y., Li, X. \& Chilkoti, A. A Modular Method for the High-Yield Synthesis of Site-Specific 867 Protein-Polymer Therapeutics. Angew Chem Int Ed Eng/ 55, 10296-10300, (2016).

86831 J., B. J., Jayanta, B. \& Ashutosh, C. A Noncanonical Function of Sortase Enables Site-Specific Conjugation 869 of Small Molecules to Lysine Residues in Proteins. Angewandte Chemie International Edition 54, 441-445, $870 \quad$ (2015).

87132 Bebis, K., Jones, M. W., Haddleton, D. M. \& Gibson, M. I. Thermoresponsive behaviour of 872 poly[(oligo(ethyleneglycol methacrylate)]s and their protein conjugates: importance of concentration and 873 solvent system. Polymer Chemistry 2, 975-982, (2011).

$87433 \quad$ Kleinert, M. et al. Animal models of obesity and diabetes mellitus. Nature Reviews Endocrinology 14, 140$875162,(2018)$.

87634 Young, A. A. et al. Glucose-lowering and insulin-sensitizing actions of exendin-4: studies in obese diabetic 877 (ob/ob, db/db) mice, diabetic fatty Zucker rats, and diabetic rhesus monkeys (Macaca mulatta). Diabetes $878 \quad 48,1026,(1999)$.

87935 Caliceti, P. \& Veronese, F. M. Pharmacokinetic and biodistribution properties of poly(ethylene glycol)880 protein conjugates. Advanced Drug Delivery Reviews 55, 1261-1277, (2003).

$88136 \quad$ Yamaoka, T., Tabata, Y. \& Ikada, Y. Distribution and tissue uptake of poly(ethylene glycol) with different 882 molecular weights after intravenous administration to mice. J Pharm Sci 83, 601-606, (1994). 
88337 Tang, D. et al. C-terminal site-specific PEGylated Exendin-4 analog: A long-acting glucagon like Peptide-1 884 receptor agonist, on glycemic control and beta cell function in diabetic db/db mice. J Pharmacol Sci 138, 885 23-30, (2018).

88638 Kanoski, S. E., Rupprecht, L. E., Fortin, S. M., De Jonghe, B. C. \& Hayes, M. R. The role of nausea in food 887 intake and body weight suppression by peripheral GLP-1 receptor agonists, exendin-4 and liraglutide. $888 \quad$ Neuropharmacology 62, 1916-1927, (2012).

88939 Ballav, C. \& Gough, S. Bydureon: long-acting exenatide for once-weekly injection. Prescriber 23, 30-33, $890 \quad$ (2012).

$89140 \quad$ Gedulin, B. R. et al. Pharmacokinetics and pharmacodynamics of exenatide following alternate routes of 892 administration. International Journal of Pharmaceutics 356, 231-238, (2008).

89341 Richter, W. F., Bhansali, S. G. \& Morris, M. E. Mechanistic determinants of biotherapeutics absorption $894 \quad$ following SC administration. Aaps j 14, 559-570, (2012).

89542 Yang, Q. \& Lai, S. K. Anti-PEG immunity: emergence, characteristics, and unaddressed questions. Wiley $896 \quad$ Interdiscip Rev Nanomed Nanobiotechnol 7, 655-677, (2015).

89743 Kozma, G. T., Shimizu, T., Ishida, T. \& Szebeni, J. Anti-PEG antibodies: Properties, formation, testing and 898 role in adverse immune reactions to PEGylated nano-biopharmaceuticals. Adv Drug Deliv Rev 154-155, 899 163-175, (2020).

$90044 \quad$ Buse, J. B. et al. Liraglutide Treatment Is Associated with a Low Frequency and Magnitude of Antibody $901 \quad$ Formation with No Apparent Impact on Glycemic Response or Increased Frequency of Adverse Events: 902 Results from the Liraglutide Effect and Action in Diabetes (LEAD) Trials. The Journal of Clinical 903 Endocrinology \& Metabolism 96, 1695-1702, (2011).

90445 Ozer, I., Tomak, A., Zareie, H. M., Baran, Y. \& Bulmus, V. Effect of Molecular Architecture on Cell 905 Interactions and Stealth Properties of PEG. Biomacromolecules 18, 2699-2710, (2017). 
90646 Richter, A. W. \& Akerblom, E. Antibodies against polyethylene glycol produced in animals by immunization 907 with monomethoxy polyethylene glycol modified proteins. Int Arch Allergy Appl Immunol 70, 124-131, 908 (1983).

90947 Stils, H. F., Jr. Adjuvants and Antibody Production: Dispelling the Myths Associated with Freund's Complete $910 \quad$ and Other Adjuvants. ILAR Journal 46, 280-293, (2005).

91148 Verhoef, J. J. F. \& Anchordoquy, T. J. Questioning the Use of PEGylation for Drug Delivery. Drug Deliv Trans/ $912 \quad \operatorname{Res} 3,499-503,(2013)$.

91349 Fathallah, A. M., Bankert, R. B. \& Balu-lyer, S. V. Immunogenicity of Subcutaneously Administered 914 Therapeutic Proteins-a Mechanistic Perspective. The AAPS Journal 15, 897-900, (2013).

$91550 \quad$ Ivens, I. A. et al. PEGylated Biopharmaceuticals: Current Experience and Considerations for Nonclinical 916 Development. Toxicol Pathol 43, 959-983, (2015).

91751 Hou, Y., Ernst, S. A., Heidenreich, K. \& Williams, J. A. Glucagon-like peptide-1 receptor is present in 918 pancreatic acinar cells and regulates amylase secretion through cAMP. Am J Physiol Gastrointest Liver $919 \quad$ Physiol 310, G26-G33, (2016).

92052 Wenande, E. \& Garvey, L. H. Immediate-type hypersensitivity to polyethylene glycols: a review. Clinical \& 921 Experimental Allergy 46, 907-922, (2016).

92253 Sellaturay, P., Nasser, S. \& Ewan, P. Polyethylene Glycol-Induced Systemic Allergic Reactions 923 (Anaphylaxis). J Allergy Clin Immunol Pract, (2020).

92454 Garvey, L. H. \& Nasser, S. Anaphylaxis to the first COVID-19 vaccine: is polyethylene glycol (PEG) the $925 \quad$ culprit? Br J Anaesth, (2020).

92655 Badiu, I., Geuna, M., Heffler, E. \& Rolla, G. Hypersensitivity reaction to human papillomavirus vaccine due 927 to polysorbate 80. BMJ Case Rep 2012, (2012).

92856 Bahar, F. G., Ohura, K., Ogihara, T. \& Imai, T. Species Difference of Esterase Expression and Hydrolase 929 Activity in Plasma. Journal of Pharmaceutical Sciences 101, 3979-3988, (2012). 
93057 Bader, M. et al. Pharmacokinetics and efficacy of PT302, a sustained-release Exenatide formulation, in a 931 murine model of mild traumatic brain injury. Neurobiol Dis 124, 439-453, (2019).

93258 He, Z. et al. Sustained release of exendin-4 from tannic acid/Fe (III) nanoparticles prolongs blood glycemic 933 control in a mouse model of type II diabetes. J Control Release 301, 119-128, (2019).

93459 Czajkowsky, D. M., Hu, J., Shao, Z. \& Pleass, R. J. Fc-fusion proteins: new developments and future 935 perspectives. EMBO Mol Med 4, 1015-1028, (2012).

93660 Mahmood, I. Application of allometric principles for the prediction of pharmacokinetics in human and 937 veterinary drug development. Advanced Drug Delivery Reviews 59, 1177-1192, (2007).

$93861 \quad$ Mann, K. V. \& Raskin, P. Exenatide extended-release: a once weekly treatment for patients with type 2 939 diabetes. Diabetes Metab Syndr Obes 7, 229-239, (2014).

94062 Bertsch, T. \& McKeirnan, K. ITCA 650. Clin Diabetes 36, 265-267, (2018). 


\section{Supplementary Files}

This is a list of supplementary files associated with this preprint. Click to download.

- SIOzeretal.pdf

- nrreportingsummaryOzeretal1.pdf 